TRANSACTIONS OF THE

AMERICAN MATHEMATICAL SOCIETY

Volume 365, Number 2, February 2013, Pages 721-766

S 0002-9947(2012)05594-2

Article electronically published on August 9, 2012

\title{
MODULI SPACE THEORY FOR THE ALLEN-CAHN EQUATION IN THE PLANE
}

\author{
MANUEL DEL PINO, MICHAŁ KOWALCZYK, AND FRANK PACARD \\ ABstract. In this paper we study entire solutions of the Allen-Cahn equation \\ $\Delta u-F^{\prime}(u)=0$, where $F$ is an even, bistable function. We are particularly \\ interested in the description of the moduli space of solutions which have some \\ special structure at infinity. The solutions we are interested in have their zero \\ set asymptotic to $2 k, k \geq 2$ oriented affine half-lines at infinity and, along each \\ of these affine half-lines, the solutions are asymptotic to the one-dimensional \\ heteroclinic solution: such solutions are called multiple-end solutions, and their \\ set is denoted by $\mathcal{M}_{2 k}$. The main result of our paper states that if $u \in \mathcal{M}_{2 k}$ \\ is nondegenerate, then locally near $u$ the set of solutions is a smooth manifold \\ of dimension $2 k$. This paper is part of a program whose aim is to classify all \\ $2 k$-ended solutions of the Allen-Cahn equation in dimension 2 , for $k \geq 2$.
}

\section{INTRODUCTION}

In this paper, we are interested in the space of solutions of some class of semilinear elliptic equations which are defined in the plane $\mathbb{R}^{2}$ (i.e. entire solutions) and whose prototype is the Allen-Cahn equation

$$
\Delta u+u-u^{3}=0 .
$$

Since we are in dimension 2, an entire solution of (1.1) which is strictly monotone in one direction is known to depend only on one variable and, after a suitable rigid motion, it has the form

$$
u_{0}(x, y):=\tanh \left(\frac{x}{\sqrt{2}}\right) .
$$

This result, originally conjectured by de Giorgi, was proven in 9 by Ghoussoub and Gui. In particular, the nodal set of a solution which is strictly monotone in one direction is given by a straight line.

In [8, the authors of the present paper, together with J. Wei, have constructed new examples of entire solutions of (1.1). Let us recall this result in more detail since it serves as a motivation for the present paper. For any $k \geq 2$, it is proven in [8] that there exist solutions of (1.1) whose nodal sets are, away from a compact set, asymptotic at infinity to $2 k$-oriented affine half-lines. Moreover, it follows from the construction in 8 , that these solutions are not isolated and in fact that they depend on a finite number of continuous parameters. Besides these, other entire solutions of (1.1) are known to exist. For example, solutions with dihedral symmetry have been constructed in [7] (see also [2, [10] and [5]).

All these solutions share the same structure at infinity; namely, their nodal set is, away from a compact set, asymptotic to a finite number of oriented affine half-lines.

Received by the editors September 6, 2010 and, in revised form, March 2, 2011.

2010 Mathematics Subject Classification. Primary 35B08, 35P99, 35Q80.

(C)2012 American Mathematical Society 
Moreover, they tend to either \pm 1 at infinity, away from a neighborhood of the nodal set where they are asymptotic to suitable translated and rotated copies of $\pm u_{0}$. In the present paper, we analyze the structure of the space of all entire solutions of (1.1) which, at infinity, are asymptotic to $2 k$ copies of $\pm u_{0}$ (modulo the action of some rigid motion). We show that, under some nondegeneracy assumption, this space is a smooth manifold whose dimension is equal to $2 k$, in agreement with the number of degrees of freedom which are available in the construction proposed in 8]. We also prove that the solutions constructed in 8] are smooth points in this space.

1.1. The heteroclinic solution. We start with a brief review of the material which is necessary both for our analysis and for a precise statement of our results. We assume that we are given a double well potential $F$. Namely, a function $F$ which is even, at least of class $\mathcal{C}^{2}$, is positive and which has only two zeros at the points \pm 1 . We further assume that the zeros of $F$ are nondegenerate minima. Hence,

$$
\begin{gathered}
F(t)>0 \text { for all } t \neq \pm 1, \quad F( \pm 1)=0, \\
F^{\prime \prime}( \pm 1) \neq 0 \text { and } F(t)=F(-t) \text { for all } t \in \mathbb{R} .
\end{gathered}
$$

It is convenient to define

$$
\alpha:=\sqrt{F^{\prime \prime}( \pm 1)}
$$

The unique solution of

$$
\ddot{u}_{0}-F^{\prime}\left(u_{0}\right)=0
$$

which tends to -1 as $x$ tends to $-\infty$, tends to +1 as $x$ tends to $+\infty$, and which is equal to 0 when $x=0$, is called the heteroclinic solution (here - denotes differentiation with respect to the variable $x$ ). It is given implicitly by the identity

$$
x=\int_{0}^{u_{0}(x)} \frac{d s}{\sqrt{2 F(s)}},
$$

which follows from the fact that

$$
\dot{u}_{0}^{2}-2 F\left(u_{0}\right)=0 .
$$

Observe that this last equality also implies that the function $u_{0}$ is strictly increasing.

Example 1.1. A typical (or classical) example is the one where the function $F$ is given by

$$
F(t)=\frac{1}{4}\left(1-t^{2}\right)^{2}
$$

in which case we have explicitly

$$
u_{0}(x)=\tanh \left(\frac{x}{\sqrt{2}}\right) .
$$

Remark 1.1. For the sake of simplicity, we have assumed that the function $F$ is even. Most of the results and techniques of the present paper do not require this assumption but, since the notation becomes quite involved in the general case, we have chosen to restrict our attention to the case where the potential $F$ is an even function. 
We collect some basic information about the spectrum of the operator

$$
L:=-\partial_{x}^{2}+F^{\prime \prime}\left(u_{0}\right),
$$

which arises as the linearized operator of (1.3) about $u_{0}$ and which is acting on functions defined on $\mathbb{R}$. All the information we need is included in the:

Lemma 1.1. The spectrum of the operator $L$ is the union of a finite or possibly infinite number of eigenvalues $\left(\mu_{j}\right)_{j \geq 0}$,

$$
\mu_{0}=0<\mu_{1}<\cdots<\mu_{n}<\cdots<\alpha^{2}:=F^{\prime \prime}( \pm 1),
$$

and the continuous spectrum which is given by $\left[\alpha^{2}, \infty\right)$.

Proof. The fact that the continuous spectrum is equal to $\left[\alpha^{2}, \infty\right)$ is standard. The fact that the bottom eigenvalue is 0 follows directly from the fact that the equation for $u_{0}$ is autonomous and hence the function $\partial_{x} u_{0}$ is in the $L^{2}$ - kernel of $L$. Since this function is positive, it has to be the eigenfunction associated to the lowest eigenvalue of $L$. Finally, the operator $L$ is of limit-point type, from which it follows that the sequence of eigenvalues is strictly increasing (we refer to [6], 3] or [23] for more details).

Remark 1.2. The number of eigenvalues of $L$ included in $\left[0, \alpha^{2}\right)$ might be finite or infinite. For notational convenience, in the case where the sequence of the eigenvalues of the operator $L$ included in $\left[0, \alpha^{2}\right)$ is finite and equal to $\mu_{0}<\mu_{1}<\cdots<\mu_{n}$, we agree that $\mu_{j}:=\alpha^{2}$, for all $j \geq n+1$. With such a convention, the sequence $\left(\mu_{j}\right)_{j \geq 0}$ is defined for all $j \geq 0$ and, if we define the Rayleigh quotient

$$
\mathcal{R}(v):=\frac{\int_{\mathbb{R}}\left(\left|\partial_{x} v\right|^{2}+F^{\prime \prime}\left(u_{0}\right) v^{2}\right) d x}{\int_{\mathbb{R}} v^{2} d x},
$$

the values of the sequence $\left(\mu_{j}\right)_{j \geq 0}$ coincide with the values of

$$
\sup _{\operatorname{dim} E=i} \inf _{v \in H^{1}(\mathbb{R})}\left\{\mathcal{R}(v): v \perp_{L^{2}} w, \quad \forall w \in E\right\}
$$

as $i \geq 0$ varies.

Example 1.2. In the case where the nonlinearity is given by (1.5), the spectrum of $L$ is explicitly known. The eigenvalues are given by

$$
\mu_{0}=0, \quad \text { with associated eigenfunction } \quad w_{0}(x)=\frac{1}{\cosh ^{2}\left(\frac{x}{\sqrt{2}}\right)},
$$

and

$$
\mu_{1}=\frac{3}{2}, \quad \text { with associated eigenfunction } \quad w_{1}(x)=\frac{\sinh \left(\frac{x}{\sqrt{2}}\right)}{\cosh ^{2}\left(\frac{x}{\sqrt{2}}\right)},
$$

while the bottom of the continuous spectrum is $\alpha^{2}=2$. For a proof of this fact, we refer to [20].

We denote by $\Pi_{j}$ the $L^{2}(\mathbb{R})$-orthogonal projection over the $j$-th eigenspace of $L$. Since the eigenspace associated to the eigenvalue 0 is spanned by the function $\partial_{x} u_{0}$, we have the explicit formula

$$
\Pi_{0}(w)=\frac{1}{\left\|\partial_{x} u_{0}\right\|_{L^{2}}^{2}}\left(\int_{\mathbb{R}} \partial_{x} u_{0} w d x\right) \partial_{x} u_{0} .
$$




\section{Statement of the Results}

In this paper, we are interested in the space of a special class of solutions of the equation

$$
\Delta u-F^{\prime}(u)=0,
$$

which are defined in $\mathbb{R}^{2}$. For example, given $r \in \mathbb{R}$ and a unit vector e $\in S^{1}$, we can define

$$
u(\mathrm{x}):=u_{0}\left(\mathrm{x} \cdot \mathrm{e}^{\perp}-r\right),
$$

where $\perp$ denotes the rotation of angle $\pi / 2$ in $\mathbb{R}^{2}$. Clearly $u$ is a solution of (2.1) and this reflects the invariance of (2.1) under the action of the group of rigid motions of $\mathbb{R}^{2}$. We say that $\pm u$ are the model solutions whose nodal set is the affine line

$$
s \longmapsto r \mathrm{e}^{\perp}+s \mathrm{e} .
$$

The class of solutions we are interested in are solutions of (2.1) which are defined in the entire space and whose nodal set is, away from some compact set, the union of $2 k$ curves which are asymptotic to $2 k$-oriented affine half-lines. Moreover, as one goes to infinity along one of these affine half-lines, the solution converges to one of the two model solutions with this affine line as nodal set. We make this definition quantitatively precise in the next paragraph. In any case, the set of all such solutions is denoted by $\mathcal{M}_{2 k}$ and the main result of this paper asserts that, under some nondegeneracy assumption, the set $\mathcal{M}_{2 k}$ is a smooth manifold of dimension $2 k$. This is a nontrivial fact: observe that in principle each of those half-lines is determined by 2 parameters, thus yielding a total of $4 k$ parameters.

2.1. Geometric description of the solutions. As promised, we give a precise description of the solutions we are interested in. This requires some preliminary definitions. At the heart of the description of the nodal set of the solutions is the set $\Lambda$ of oriented affine lines in $\mathbb{R}^{2}$. Any element $\lambda \in \Lambda$ can be uniquely written as

$$
\lambda:=r \mathrm{e}^{\perp}+\mathbb{R} \mathrm{e},
$$

for some $r \in \mathbb{R}$ and some unit vector e $\in S^{1}$, which defines the orientation of the line. Recall that we denote by $\perp$ the rotation of angle $\pi / 2$ in $\mathbb{R}^{2}$. Clearly, $\Lambda$ is diffeomorphic to $\mathbb{R} \times S^{1}$ and writing $\mathrm{e}=(\cos \theta, \sin \theta)$, we get local coordinates $(r, \theta)$ in $\Lambda$. Observe that the affine lines are oriented and hence we do not identify the line corresponding to $(r, \theta)$ and the line corresponding to $(-r, \theta+\pi)$. There is also a natural symplectic structure on $\Lambda$ which, in these local coordinates, is given by

$$
\omega:=d r \wedge d \theta \text {. }
$$

Note that the map $J$ defined by

$$
J \partial_{\theta}=-\partial_{r} \quad \text { and } \quad J \partial_{r}=\partial_{\theta}
$$

(which corresponds to the rotation by $\pi / 2$ in the tangent space) induces an almost complex structure on $\Lambda$. This map, together with the 2 -form $\omega$ induces the natural metric on $\Lambda$,

$$
g=d r^{2}+d \theta^{2} .
$$

More generally, for all $k^{\prime} \geq 1$, let us denote by $\Lambda^{k^{\prime}}$ the set of $k^{\prime}$-tuples of oriented affine lines in $\mathbb{R}^{2}$. This set is clearly diffeomorphic to $\mathbb{R}^{k^{\prime}} \times\left(S^{1}\right)^{k^{\prime}}$ and, 
again, there exists a natural symplectic structure on $\Lambda^{k^{\prime}}$ which, in local coordinates $\left(r_{1}, \ldots, r_{k^{\prime}}, \theta_{1}, \ldots, \theta_{k^{\prime}}\right)$, can be written as

$$
\omega_{k^{\prime}}:=d r_{1} \wedge d \theta_{1}+\cdots+d r_{k^{\prime}} \wedge d \theta_{k^{\prime}} .
$$

The almost complex structure and the metric on $\Lambda^{k^{\prime}}$ can be introduced in the same way as was done for $\Lambda$.

We have the definition:

Definition 2.1. A $k^{\prime}$-tuple of oriented affine lines $\lambda=\left(\lambda_{1}, \ldots, \lambda_{k^{\prime}}\right) \in \Lambda^{k^{\prime}}$ is said to be ordered if each $\lambda_{j}$ can be written as

$$
\lambda_{j}:=r_{j} \mathrm{e}_{j}^{\perp}+\mathbb{R} \mathrm{e}_{j},
$$

for some $r_{j} \in \mathbb{R}$ and some unit vector $\mathrm{e}_{j} \in S^{1}$, which can be written as $\mathrm{e}_{j}=$ $\left(\cos \theta_{j}, \sin \theta_{j}\right)$ with

$$
\theta_{1}<\theta_{2}<\cdots<\theta_{k^{\prime}}<2 \pi+\theta_{1} .
$$

We denote by $\Lambda_{\text {ord }}^{k^{\prime}}$ the set of $k^{\prime}$-tuples of ordered, oriented affine lines and we denote by

$$
\theta_{\lambda}:=\frac{1}{2} \min \left\{\theta_{2}-\theta_{1}, \ldots, \theta_{k^{\prime}}-\theta_{k^{\prime}-1}, 2 \pi+\theta_{1}-\theta_{k^{\prime}}\right\}
$$

the half of the minimum of the angles between any two consecutive oriented affine lines $\lambda_{1}, \ldots, \lambda_{k^{\prime}}$.

Assume that we are given a $k^{\prime}$-tuple of oriented affine lines $\lambda=\left(\lambda_{1}, \ldots, \lambda_{k^{\prime}}\right)$ as in (2.3). It is easy to check that for all $R>0$ large enough and for all $j=1, \ldots, k^{\prime}$, there exists $s_{j} \in \mathbb{R}$ such that:

(i) The point $\mathrm{x}_{j}:=r_{j} \mathrm{e}_{j}^{\perp}+s_{j} \mathrm{e}_{j}$ belongs to the circle $\partial B_{R}$, with $R>0$.

(ii) The half-lines

$$
\lambda_{j}^{+}:=\mathrm{x}_{j}+\mathbb{R}^{+} \mathrm{e}_{j}
$$

are disjoint and included in $\mathbb{R}^{2} \backslash B_{R}$.

(iii) The minimum of the distance between two distinct half-lines $\lambda_{i}^{+}$and $\lambda_{j}^{+}$is larger than 4 .

The set of affine half-lines $\lambda_{1}^{+}, \ldots, \lambda_{k^{\prime}}^{+}$together with the circle $\partial B_{R}$ induces a decomposition of $\mathbb{R}^{2}$ into $k^{\prime}+1$ slightly overlapping connected components

$$
\mathbb{R}^{2}=\Omega_{0} \cup \Omega_{1} \cup \ldots \cup \Omega_{k^{\prime}},
$$

where

$$
\Omega_{0}:=B_{R+1},
$$

and where, for $j=1, \ldots, k^{\prime}$,

$$
\Omega_{j}:=\left\{\mathrm{x} \in \mathbb{R}^{2}:|\mathrm{x}|>R-1 \quad \text { and } \operatorname{dist}\left(\mathrm{x}, \lambda_{j}^{+}\right)<\operatorname{dist}\left(\mathrm{x}, \lambda_{i}^{+}\right)+2, \quad \forall i \neq j\right\},
$$

where $\operatorname{dist}\left(\mathrm{x}, \lambda_{j}\right)$ denotes the distance to $\lambda_{j}^{+}$. Observe that, for all $j=1, \ldots, k^{\prime}$, the set $\Omega_{j}$ contains the half-line $\lambda_{j}^{+}$. 
We define $\mathbb{I}_{0}, \mathbb{I}_{1}, \ldots, \mathbb{I}_{k^{\prime}}$, a smooth partition of unity of $\mathbb{R}^{2}$ which is subordinate to the above decomposition of $\mathbb{R}^{2}$. Hence

$$
\sum_{j=0}^{k^{\prime}} \mathbb{I}_{j} \equiv 1,
$$

and the support of $\mathbb{I}_{j}$ is included in $\Omega_{j}$, for $j=0, \ldots, k^{\prime}$. Without loss of generality, we can also assume that $\mathbb{I}_{0} \equiv 1$ in

$$
\Omega_{0}^{\prime}:=B_{R-1},
$$

and $\mathbb{I}_{j} \equiv 1$ in

$$
\Omega_{j}^{\prime}:=\left\{\mathrm{x} \in \mathbb{R}^{2}:|\mathrm{x}|>R+1 \quad \text { and } \quad \operatorname{dist}\left(\mathrm{x}, \lambda_{j}^{+}\right)<\operatorname{dist}\left(\mathrm{x}, \lambda_{i}^{+}\right)-2, \quad \forall i \neq j\right\},
$$

for $j=1, \ldots, k^{\prime}$. Finally, we assume that

$$
\left\|\mathbb{I}_{j}\right\|_{\mathcal{C}^{2}\left(\mathbb{R}^{2}\right)} \leq C .
$$

We now take $k^{\prime}=2 k$, for some $k \geq 1$ and

$$
\lambda=\left(\lambda_{1}, \ldots, \lambda_{2 k}\right) \in \Lambda_{\text {ord }}^{2 k},
$$

we write $\lambda_{j}^{+}=\mathrm{x}_{j}+\mathbb{R}^{+} \mathrm{e}_{j}$ and we define

$$
u_{\lambda}:=\sum_{j=1}^{2 k}(-1)^{j} \mathbb{I}_{j} u_{0}\left(\operatorname{dist}^{s}\left(\cdot, \lambda_{j}\right)\right),
$$

where

$$
\operatorname{dist}^{s}\left(\mathrm{x}, \lambda_{j}\right):=\left(\mathrm{x}-\mathrm{x}_{j}\right) \cdot \mathrm{e}_{j}^{\perp}
$$

denotes the signed distance from a point $\mathrm{x} \in \mathbb{R}^{2}$ to $\lambda_{j}$.

Observe that, by construction, the function $u_{\lambda}$ is, away from a compact set, asymptotic to copies of the model solutions whose nodal set consists of the affine half-lines $\lambda_{1}^{+}, \ldots, \lambda_{2 k}^{+}$. A simple computation shows that $u_{\lambda}$ is not far from being a solution of (2.1) in the sense that $\Delta u_{\lambda}-F^{\prime}\left(u_{\lambda}\right)$ is a function which decays exponentially to 0 at infinity (this uses the fact that $\theta_{\lambda}>0$ ).

We are interested in solutions of (2.1) which are asymptotic to a function $u_{\lambda}$ for some choice of $\lambda \in \Lambda_{\text {ord }}^{2 k}$. More precisely, we have the:

Definition 2.2. Let $\mathcal{S}_{2 k}$ denote the set of functions $u$ which are defined in $\mathbb{R}^{2}$ and which satisfy

$$
u-u_{\lambda} \in W^{2,2}\left(\mathbb{R}^{2}\right),
$$

for some $\lambda \in \Lambda_{\text {ord }}^{2 k}$. We also define the decomposition operator $\mathcal{J}$ by

$$
\begin{aligned}
\mathcal{J}: \mathcal{S}_{2 k} & \longrightarrow W^{2,2}\left(\mathbb{R}^{2}\right) \times \Lambda_{\text {ord }}^{2 k}, \\
u & \longmapsto\left(u-u_{\lambda}, \lambda\right) .
\end{aligned}
$$

The topology on $\mathcal{S}_{2 k}$ is the one for which the operator $\mathcal{J}$ is continuous (the target space being endowed with the product topology). We define $\mathcal{M}_{2 k}$ to be the set of solutions $u$ of (2.1) which belong to $\mathcal{S}_{2 k}$. 
The existence of the heteroclinic solution $u_{0}$ shows that $\mathcal{M}_{2}$ is nonempty. Indeed, it is enough to consider

$$
u(\mathrm{x})=u_{0}\left(\mathrm{x} \cdot \mathrm{e}^{\perp}-r\right),
$$

for any unit vector e and any $r \in \mathbb{R}$. As we already discussed in the introduction, the result of [8] provides infinitely many solutions of (2.1) whose nodal set decomposes into $2 k$ nearly parallel half-lines in the case where the potential $F$ is given by (1.5). This result, together with the results in [7] and [2], implies that $\mathcal{M}_{2 k} \neq \emptyset$ for any $k \geq 1$. Investigation of the structure of $\mathcal{M}_{2 k}$ is then a natural question. In our opinion, the questions which are relevant are the following:

(1) Is the space $\mathcal{M}_{2 k}$ a smooth submanifold of $\mathcal{S}_{2 k}$ ? If so, what is the dimension of $\mathcal{M}_{2 k}$ ?

(2) There exists a natural map

$$
\mathcal{P}: \mathcal{M}_{2 k} \longrightarrow \Lambda_{\text {ord }}^{2 k}
$$

defined by

$$
\mathcal{P}(u):=\lambda,
$$

if $u-u_{\lambda} \in W^{2,2}\left(\mathbb{R}^{2}\right)$. What can be said about this map? Is it surjective? If not, can one characterize its image? In other words, what sets of affine half-lines are asymptotic to nodal sets of solutions of (2.1)?

2.2. The results. We keep the notation introduced above. Given $k \geq 1$ and

$$
\lambda=\left(\lambda_{1}, \ldots, \lambda_{2 k}\right) \in \Lambda_{\text {ord }}^{2 k},
$$

we write $\lambda_{j}^{+}=\mathrm{x}_{j}+\mathbb{R}^{+} \mathrm{e}_{j}$ as in (2.4). We denote by $\Omega_{0}, \ldots, \Omega_{2 k}$ the decomposition of $\mathbb{R}^{2}$ associated to this $2 k$-tuple of affine half-lines and by $\mathbb{I}_{0}, \ldots, \mathbb{I}_{2 k}$ the partition of unity subordinate to this partition. Given $\gamma, \delta \in \mathbb{R}$, we define a weight function $\Gamma_{\gamma, \delta}$ such that

in $\Omega_{0}$ and, for $j=1, \ldots, 2 k$,

$$
\Gamma_{\gamma, \delta}(\mathrm{x}) \sim 1
$$

$$
\Gamma_{\gamma, \delta}(\mathrm{x}) \sim e^{\gamma s}(\cosh r)^{\delta}
$$

in $\Omega_{j}$, where we have written

$$
\mathrm{x}=\mathrm{x}_{j}+r \mathrm{e}_{j}^{\perp}+s \mathrm{e}_{j},
$$

for some $r \in \mathbb{R}$ and $s>0$ (observe that $(r, s)$ are local coordinates which are well defined in each $\Omega_{j}$ ). As usual, the notation $f \sim g$ means that there exists some constant $C>1$ such that

$$
\frac{1}{C}|g| \leq|f| \leq C|g|
$$

The explicit definition of the weight function $\Gamma_{\gamma, \delta}$ is given by

$$
\Gamma_{\gamma, \delta}(\mathrm{x}):=\mathbb{I}_{0}(\mathrm{x})+\sum_{j=1}^{2 k} \mathbb{I}_{j}(\mathrm{x}) e^{\gamma\left(\mathrm{x}-\mathrm{x}_{j}\right) \cdot \mathrm{e}_{j}}\left(\cosh \left(\left(\mathrm{x}-\mathrm{x}_{j}\right) \cdot \mathrm{e}_{j}^{\perp}\right)\right)^{\delta}
$$

so that, by construction, $\gamma$ is the rate of decay or blowup along the half-lines $\lambda_{j}^{+}$ and $\delta$ is the rate of decay or blowup in the direction orthogonal to $\lambda_{j}^{+}$.

With this definition in mind, we define the weighted Lebesgue space

$$
L_{\gamma, \delta}^{2}\left(\mathbb{R}^{2}\right):=\Gamma_{\gamma, \delta} L^{2}\left(\mathbb{R}^{2}\right)
$$


and the weighted Sobolev space

$$
W_{\gamma, \delta}^{2,2}\left(\mathbb{R}^{2}\right):=\Gamma_{\gamma, \delta} W^{2,2}\left(\mathbb{R}^{2}\right) .
$$

Observe that, even though this does not appear in the notation, the partition of unity, the weight function and the induced weighted spaces all depend on the choice of $\lambda \in \Lambda_{\text {ord }}^{2 k}$.

Our first result shows that, if $u$ is a solution of (2.1) which is close to $u_{\lambda}$ (in the $W^{2,2}$ topology), then $u-u_{\lambda}$ tends to 0 exponentially fast at infinity.

Theorem 2.1 (Refined asymptotics). Assume that $u \in \mathcal{S}_{2 k}$ is a solution of (2.1) and define $\lambda=\mathcal{P}(u) \in \Lambda_{\text {ord }}^{2 k}$, so that

$$
u-u_{\lambda} \in W^{2,2}\left(\mathbb{R}^{2}\right) .
$$

Then, there exist $\delta \in(0, \alpha)$ and $\gamma>0$ such that

$$
u-u_{\lambda} \in W_{-\gamma,-\delta}^{2,2}\left(\mathbb{R}^{2}\right) .
$$

More precisely, $\delta>0$ and $\gamma>0$ can be chosen so that

$$
\gamma \in\left(0, \sqrt{\mu_{1}}\right), \quad \gamma^{2}+\delta^{2}<\alpha^{2} \quad \text { and } \quad \alpha>\delta+\gamma \cot \theta_{\lambda},
$$

where $\theta_{\lambda}$ is equal to the half of the minimum of the angles between two consecutive oriented affine lines $\lambda_{1}, \ldots, \lambda_{2 k}$ (see Definition 2.1).

In particular, this implies that, given $u \in \mathcal{M}_{2 k}$, there exists $\bar{\delta}>0$ such that

$$
\mathcal{J}(u) \in e^{-\bar{\delta}|\mathbf{x}|^{2}} W^{2,2}\left(\mathbb{R}^{2}\right) \times \Lambda_{\text {ord }}^{2 k} .
$$

It will be clear from the proof of this result that more is true. Namely, the choice of $\bar{\delta}$ can be made uniform in any neighborhood of $u \in \mathcal{M}_{2 k}$ in $\mathcal{S}_{2 k}$. More, precisely, given $u \in \mathcal{M}_{2 k}$, there exists $\delta_{u}>0$ and there exists a neighborhood $\mathcal{U}$ of $u$ in $\mathcal{S}_{2 k}$ such that

$$
\begin{array}{ccc}
\mathcal{J}: \mathcal{U} \cap \mathcal{M}_{2 k} & \longrightarrow & e^{-\delta_{u}|x|^{2}} W^{2,2}\left(\mathbb{R}^{2}\right) \times \Lambda_{\text {ord }}^{2 k} \\
\bar{u} & \longmapsto & \mathcal{J} \bar{u},
\end{array}
$$

is well defined and continuous (observe that continuity of this mapping is not a straightforward consequence of the definition of $\mathcal{J}$ ).

Before we state the next result, we have to introduce the notion of nondegeneracy in this context.

Definition 2.3. A function $u \in \mathcal{M}_{2 k}$ is said to be nondegenerate if the linearized operator

$$
-\Delta+F^{\prime \prime}(u)
$$

is injective in the space $L_{-\gamma, \delta}^{2}\left(\mathbb{R}^{2}\right)$, for some $\gamma \in\left(0, \sqrt{\mu}_{1}\right)$ and some $\delta \in \mathbb{R}$ satisfying

$$
\gamma^{2}+\delta^{2}<\alpha^{2} .
$$

As already mentioned, the existence of a family of solutions of (2.1) which belongs to $\mathcal{M}_{2 k}$ is guaranteed by the result in 8$]$, for the equation

$$
\Delta u+u-u^{3}=0 .
$$


We prove in $\$ 9$ that the solutions obtained in [8] are nondegenerate; this implies that:

Proposition 2.1. In the case where the nonlinearity is given by $F(u)=\frac{1}{4}\left(1-u^{2}\right)^{2}$ (and the equation is given by (2.15)), for each $k \geq 1, \mathcal{M}_{2 k}$ contains nondegenerate elements.

For the sake of simplicity, when proving Proposition 2.1. we restrict our attention to the special nonlinearity

$$
F(u)=\frac{1}{4}\left(1-u^{2}\right)^{2}
$$

since the existence of solutions is proven for this special nonlinearity in (2.15). The method used in 8 extends in a straightforward manner to nonlinear equations of the type (2.1) and hence, it can be shown that for general nonlinearities, $\mathcal{M}_{2 k}$ also contains nondegenerate elements.

Checking whether a given solution is nondegenerate or not is a hard problem. For example, in [7, a solution that belongs to $\mathcal{M}_{4}$ is built for the nonlinearity (1.5). Since its nodal set is the union of two perpendicular lines it is known as the saddle solution. The proof of the fact that this solution is nondegenerate is given in [14].

The second result of this paper is the following:

Theorem 2.2 (Dimension of the moduli space). Assume that $u \in \mathcal{M}_{2 k}$ is nondegenerate. Then, in a neighborhood of $u$ in $\mathcal{S}_{2 k}$, the set of solutions of (2.1) is a smooth manifold of dimension $2 k$.

Near any nondegenerate elements of $\mathcal{M}_{2 k}$, we also have some information about the mapping $\mathcal{P}$.

Theorem 2.3. Assume that $u \in \mathcal{S}_{2 k}$ is nondegenerate. Then, there exists an open neighborhood of $u$ in $\mathcal{S}_{2 k}$ whose image by $\mathcal{P}$ is a Lagrangian submanifold of $\Lambda^{2 k}$ for the symplectic structure defined in (2.2).

Geometrically the meaning of the mapping $\mathcal{P}$ is clear: Given any solution $u \in$ $\mathcal{M}_{2 k}, \mathcal{P}(u) \in \Lambda^{2 k}$ corresponds to the choice of $2 k$ oriented affine lines which determine the asymptotics of the nodal set of $u$ at infinity. Theorem 2.2 and Theorem 2.3 show that there is in reality less freedom than what might be initially expected in selecting the half-lines which are the asymptotes of the nodal sets of the solutions of (2.1). Indeed, at regular points of $\mathcal{M}_{2 k}$, the image of $\mathcal{P}$ is a $2 k$-dimensional submanifold of $\Lambda^{2 k}$ which is $4 k$-dimensional.

Observe that the image of $\mathcal{P}$ is naturally constrained. In fact, if $u \in \mathcal{M}_{2 k}$ and

$$
\mathcal{P}(u)=\left(\lambda_{1}, \ldots, \lambda_{2 k}\right),
$$

with

it follows from [10] that

$$
\lambda_{j}=r_{j} \mathrm{e}_{j}^{\perp}+\mathbb{R} \mathrm{e}_{j},
$$

$$
\sum_{j=1}^{2 k} \mathrm{e}_{j}=0
$$

Moreover, pushing further the analysis in [10, we can also prove that

$$
\sum_{j=1}^{2 k} r_{j}=0 .
$$


We refer to these equalities as the balancing formula for the Allen-Cahn equation and, for the sake of completeness, we give a simple proof of these equalities in Appendix A. Observe that (2.16) implies that the angle between two consecutive half-lines is always less than or equal to $\pi$ and that it can only be equal to $\pi$ when $k=1$. Therefore, if $u \in \mathcal{M}_{2 k}$ and $\lambda=\mathcal{P}(u)$, we always have

$$
0 \leq \theta_{\lambda} \leq \pi / 2 .
$$

The proofs of our results follow from the application of the implicit function theorem in a suitably designed weighted function space. The results and the arguments are very much in the spirit of what has already been done in the study of the moduli spaces of complete noncompact constant mean curvatures surfaces in Euclidean space or complete constant scalar curvature metrics in conformal geometry; see [15], 18] and [17].

2.3. Comments and open problems. The previous results raise some interesting questions and comments. The barrier construction in [7] generalizes to yield, for each $k \geq 2$, a solution with dihedral symmetry whose nodal set is given by the union of the oriented half-lines

$$
\lambda_{j}^{+}=\mathbb{R}^{+}(\cos (j \pi / k), \sin (j \pi / k)),
$$

for $j=1, \ldots, 2 k$. These solutions, whose existence is proven in [2, [10] and [5, are referred to as $k$-th saddle solutions. In [14, it is proven that the saddle solution is nondegenerate when $k=1$. Is it true that, for each $k \geq 2$, the $k$-th saddle solutions are nondegenerate in the sense of Definition 2.3?

Proving this type of result would provide strong evidence that, given $k \geq 2$, the solutions with $k$ nearly parallel nodal lines constructed in [8], and the $k$-th saddle solutions belong to the same connected component of $\mathcal{M}_{2 k}$.

Obviously, (2.1) is invariant under the action of translations and rotations of the plane. When $k=2$, Theorem 2.3 implies that, near any nondegenerate element, $\mathcal{M}_{4}$ is 4-dimensional, but rigid motions already yield 3 degrees of freedom; therefore, there is only one free parameter which does not come from the action of rigid motions. This is in agreement with the result in [8], where solutions of (2.1) which are even under symmetry with respect to both the $x$-axis and the $y$-axis are constructed. It is proven in [11] that all elements of $\mathcal{M}_{4}$ are even with respect to two perpendicular lines. When $k=2$ one should be able to prove that $\mathcal{M}_{4}$ has only one connected component (we agree that we identify the components corresponding to $u$ and $-u$ ). Let us mention that, when $k \geq 3$, solutions without any symmetries have been constructed in 8$]$.

There are no examples of two distinct solutions of (2.1) whose nodal sets are asymptotic to the same set of affine half-lines at infinity. Is it true that the mapping $\mathcal{P}$ is injective? Assuming that any element of $\mathcal{M}_{2 k}$ is nondegenerate, the image of $\mathcal{M}_{2 k}$ by the mapping $\mathcal{P}$ would then be an embedded Lagrangian submanifold in $\Lambda^{2 k}$.

Finally, to end this introduction, we briefly describe the plan of the paper. We start in $\S 3$ with the analysis of the mapping properties of the family of second-order linear operators

$$
L_{\zeta}:=-\partial_{x}^{2}+F^{\prime \prime}\left(u_{0}\right)-\zeta^{2}
$$

which depend on a complex parameter $\zeta \in \mathbb{C}$. Here $-\partial_{x}^{2}+F^{\prime \prime}\left(u_{0}\right)$ is the linearized operator about the heteroclinic solution $u_{0}$. This analysis reduces to the 
analysis of a second-order linear ordinary differential equation which is standard. However, the original feature is that this operator is acting on weighted spaces $(\cosh x)^{\delta} W^{2,2}(\mathbb{R}, \mathbb{C})$. Depending on $\zeta \in \mathbb{C}$ and $\delta \in \mathbb{R}$, we study the surjectivity and injectivity properties of $L_{\zeta}$. We also provide some estimates for the inverse of this operator (when it is well defined).

The analysis of $\S 3$, together with Fourier analysis, allows us to derive in $\S 4$ the mapping properties of

$$
\mathcal{L}:=-\Delta+F^{\prime \prime}\left(u_{0}\right),
$$

which is the linearized operator about the model solution. Again, we are interested in the mapping properties of $\mathcal{L}$ when it is acting on the weighted space $e^{\gamma y}(\cosh x)^{\delta} W^{2,2}\left(\mathbb{R}^{2}\right)$, where $\delta, \gamma \in \mathbb{R}$. The main result is the selection of the range of weights $\delta$ and $\gamma$ for which the operator is an isomorphism. This section is concluded by what is usually referred to as the Linear Decomposition Lemma. In essence, it states that, if $\mathcal{L} w=v$ where both $w$ and $v$ belong to weighted spaces and if $v$ belongs to a smaller weighted space, then the function $w$ has a nice decomposition at infinity. This section parallels the corresponding analysis for some class of elliptic operators defined on manifolds with cylindrical ends which is done in [16] (see also 22] for a concise exposition of some of these ideas).

In $\S 5$, we use the results of the previous section to extend this analysis to the analysis of the operator

$$
\mathfrak{L}:=-\Delta+F^{\prime \prime}(u),
$$

where $u$ is any element of $\mathcal{M}_{2 k}$. Again we define an appropriate weighted space and study the mapping properties of the operator $\mathfrak{L}$ acting on these spaces. Roughly speaking the weighted spaces are obtained by gluing together the weighted spaces defined in $\S 3$ along each end of $u$. Again, we identify the range of weights for which $\mathfrak{L}$ is Fredholm. In the next section, $\S 6$, we derive a Linear Decomposition Lemma for the operator $\mathfrak{L}$.

In $\S 7$, we prove the Refined Asymptotics Theorem (Theorem 2.1). The proof makes extensive use of the Linear Decomposition Lemma together with a scaling argument which is originally due to L. Simon in a different context and which was used in $[12$.

Finally, in $\S 8$, we set up the correct framework in which the implicit function theorem can be applied to prove Theorem 2.2. Again, the linear analysis of $\S 5$ and the Linear Decomposition Lemma in $\$ 6$ are crucial ingredients of the proof. This part borrows ideas which have been used for example in [15] and which are by now classical. Section 9 is devoted to the proof of Proposition 2.1 and, in the last section, $\S 10$, we give a proof of Theorem 2.3 which roughly speaking is a consequence of the Linear Decomposition Lemma and an integration by parts.

\section{THE LINEARIZED OPERATOR ABOUT THE HETEROCLINIC SOLUTION}

We consider the heteroclinic solution $u_{0}$ which has been defined in $\S 1$ and we recall that we have already defined the operator

$$
L:=-\partial_{x}^{2}+F^{\prime \prime}\left(u_{0}\right) .
$$

To simplify notation, we set

$$
S:=\left\{ \pm \sqrt{\mu}_{j}: j \in \mathbb{N}\right\}
$$


where the $\left(\mu_{j}\right)_{j \geq 0}$ are the eigenvalues of $L$ (which form a finite or infinite sequence) and where we recall that we have defined

$$
\alpha:=\sqrt{F^{\prime \prime}( \pm 1)} .
$$

Given any complex number $\zeta:=\gamma+i \xi \in \mathbb{C}$, with $\gamma, \xi \in \mathbb{R}$, we define

$$
L_{\zeta}:=L-\zeta^{2},
$$

and we define the complex number $\eta:=\delta+i \mu \in \mathbb{C}$, with $\delta \geq 0$ by the identity

$$
\eta^{2}=\alpha^{2}-\zeta^{2} \text {. }
$$

Observe that we have

$$
\delta^{2}=\alpha^{2}-\gamma^{2}+\xi^{2}+\mu^{2} \geq \alpha^{2}-\gamma^{2} .
$$

The following result classifies the behavior of the solutions of the homogeneous problem $L_{\zeta} W=0$ at $\pm \infty$.

Lemma 3.1. There exist two linearly independent (complex-valued) functions $W_{\zeta}^{ \pm}$, which are solutions of $L_{\zeta} W_{\zeta}^{ \pm}=0$ in $\mathbb{R}$, such that:

(i) If $\zeta \neq \pm \alpha$, then $W_{\zeta}^{ \pm}(x) \sim e^{ \pm \eta x}$, as $x$ tends to $+\infty$.

(ii) If $\zeta= \pm \alpha$, then $W_{\zeta}^{+}(x) \sim 1$ and $W_{\zeta}^{-}(x) \sim x$, as $x$ tends to $+\infty$.

Proof. We give the proof of the result when $\zeta \neq \pm \alpha$; the other case can be treated using similar arguments. When $\zeta \neq \pm \alpha$, one simply uses the fact that

$$
\left(\partial_{x}^{2}-\alpha^{2}+\zeta^{2}\right) e^{ \pm \eta x}=0
$$

together with the fact that $u_{0}$ tends to 1 exponentially fast at $+\infty$. The existence of $W_{\zeta}^{ \pm}$follows at once from a standard perturbation argument for ordinary differential equations.

Thanks to the previous result, we can define the indicial roots of the operator $L_{\zeta}$ which characterize the asymptotic behavior of solutions of the homogeneous problem $L_{\zeta} w=0$.

Definition 3.1. The indicial roots of $L_{\zeta}$ are the real numbers defined by

$$
\pm \delta_{\zeta}:= \pm \Re \sqrt{\alpha^{2}-\zeta^{2}}
$$

Observe that

$$
\delta_{\bar{\zeta}}=\delta_{\zeta}
$$

The following simple observation is crucial:

Lemma 3.2. The following properties hold:

(i) Assume that $\gamma \in[-\alpha, \alpha]$. Then

$$
\min _{\xi \in \mathbb{R}} \delta_{\gamma+i \xi}=\sqrt{\alpha^{2}-\gamma^{2}} .
$$

(ii) Assume that $|\gamma| \geq \alpha$. Then

$$
\min _{\xi \in \mathbb{R}} \delta_{\gamma+i \xi}=0 .
$$

Proof. This easily follows from (3.2). 
Given $\delta \in \mathbb{R}$, we define the weighted space

$$
\tilde{L}_{\delta}^{2}(\mathbb{R}, \mathbb{C}):=(\cosh x)^{\delta} L^{2}(\mathbb{R}, \mathbb{C}),
$$

which is equipped with the natural norm

$$
\|W\|_{\tilde{L}_{\delta}^{2}(\mathbb{R}, \mathbb{C})}:=\left\|(\cosh x)^{-\delta} W\right\|_{L^{2}(\mathbb{R}, \mathbb{C})} .
$$

We consider the unbounded operator

$$
\begin{aligned}
A_{\zeta, \delta}: \quad \tilde{L}_{\delta}^{2}(\mathbb{R}, \mathbb{C}) & \longmapsto \tilde{L}_{\delta}^{2}(\mathbb{R}, \mathbb{C}), \\
W & \longmapsto L_{\zeta} W .
\end{aligned}
$$

The subscripts $\zeta, \delta$ keep track of the weights $\delta$ and the complex number $\zeta$ which appears in $L_{\zeta}$ and the parameter $\delta$ which appears in the definition of $\tilde{L}_{\delta}^{2}(\mathbb{R}, \mathbb{C})$. It is easy to check that the operator $A_{\zeta, \delta}$ is an unbounded operator with dense domain equal to

$$
\tilde{W}_{\delta}^{2,2}(\mathbb{R}, \mathbb{C}):=(\cosh x)^{\delta} W^{2,2}(\mathbb{R}, \mathbb{C}),
$$

and also that it has a closed graph. The following result is by now well known and follows for example from [16] (see also [22]):

Proposition 3.1. Assume that $\delta_{\zeta}>0$ and further assume that the operator $A_{\zeta, \delta}$ is injective for some $\delta \in\left(-\delta_{\zeta}, \delta_{\zeta}\right)$. Then $A_{\zeta, \delta}$ is an isomorphism for any $\delta \in\left(-\delta_{\zeta}, \delta_{\zeta}\right)$.

Proof. As mentioned, the proof of this result follows from the general theory developed in 16] or also in 22. Since we are dealing with a second-order ordinary differential equation, a direct proof is also available. We give here the two proofs.

First proof. Using the standard Hermitian product on $L^{2}(\mathbb{R}, \mathbb{C})$, we can identify the dual of $\tilde{L}_{\delta}^{2}(\mathbb{R}, \mathbb{C})$ with $\tilde{L}_{-\delta}^{2}(\mathbb{R}, \mathbb{C})$. With this identification, the adjoint of $A_{\zeta, \delta}$ can be identified with $A_{\bar{\zeta},-\delta}$, since

$$
\int_{\mathbb{R}} L_{\zeta} W \bar{V} d x=\int_{\mathbb{R}} W \overline{L_{\bar{\zeta}} V} d x
$$

for all smooth, complex-valued functions $V$ and $W$ having compact support. Let us assume $\delta$ is not an indicial root of $L_{\zeta}$ (namely $\delta \neq \pm \delta_{\zeta}$ ). In this case, it follows from [16] or 22] that the operator $A_{\zeta, \delta}$ is Fredholm and has closed range. Moreover, this operator is injective if and only if $A_{\bar{\zeta},-\delta}$ is surjective.

Now, let us assume that $\delta<\delta_{\zeta}$. Using Lemma 3.1, we check that any solution of $L_{\zeta} W=0$ which is bounded by $(\cosh x)^{\delta}$ is in fact bounded by $(\cosh x)^{-\delta_{\zeta}}$. Hence, if the operator $A_{\zeta, \delta}$ is injective for some $\delta \in\left(-\delta_{\zeta}, \delta_{\zeta}\right)$, it is then injective for any $\delta \in\left(-\delta_{\zeta}, \delta_{\zeta}\right)$. Finally, assume that $\delta \in\left(-\delta_{\zeta}, \delta_{\zeta}\right)$ and that $A_{\zeta, \delta}$ is injective. Then, $A_{\bar{\zeta},-\delta}$ is also injective and, by the above duality argument, $A_{\zeta, \delta}$ is also surjective. This completes the proof of the result.

Second proof. We assume that $\delta_{\zeta}>0$ (otherwise there is nothing to prove) and we choose $\delta \in\left(-\delta_{\zeta}, \delta_{\zeta}\right)$. The space of solutions of the homogeneous problem $L_{\zeta} W=0$ is spanned by the two functions $W_{\zeta}^{ \pm}$which have been defined in Lemma 3.1 By construction $W_{\zeta}^{-}(x) \sim e^{-\eta x}$ at $+\infty$ and hence it is bounded by a constant times $(\cosh x)^{\delta}$ at $+\infty$. Since we assume that $A_{\zeta, \delta}$ is injective, then necessarily we also have $W_{\zeta}^{-}(x) \sim e^{-\eta x}$ at $-\infty$. Without loss of generality, we can assume that $W_{\zeta}^{+}$ is constructed in such a way that $W_{\zeta}^{+}(x) \sim e^{\eta x}$ at $\pm \infty$. 
Let us denote by $\mathbb{W}_{\zeta}$ the Wronskian of $W_{\zeta}^{ \pm}$. Namely,

$$
\mathbb{W}_{\zeta}:=W_{\zeta}^{+} \partial_{x} W_{\zeta}^{-}-W_{\zeta}^{-} \partial_{x} W_{\zeta}^{+} .
$$

Given $V \in \tilde{L}_{\delta}^{2}(\mathbb{R}, \mathbb{C})$, it is easy to check that

$$
W(x):=\frac{1}{\mathbb{W}_{\zeta}}\left(W_{\zeta}^{-}(x) \int_{-\infty}^{x} W_{\zeta}^{+} V d x+W_{\zeta}^{+}(x) \int_{x}^{+\infty} W_{\zeta}^{-} V d x\right)
$$

is well defined (this uses the fact that $\delta<\delta_{\zeta}$ ) and is a solution of $L_{\zeta} W=V$. Moreover, direct estimates imply that $W \in \tilde{L}_{\delta}^{2}(\mathbb{R}, \mathbb{C})$ and also that

$$
\|W\|_{\tilde{L}_{\delta}^{2}(\mathbb{R}, \mathbb{C})} \leq C\|V\|_{\tilde{L}_{\delta}^{2}(\mathbb{R}, \mathbb{C})},
$$

for some constant $C>0$ independent of $V$. Details of the derivation of this estimate are postponed to Appendix B. This completes the proof of the result.

The main result of this section is the following:

Proposition 3.2. Assume that $\gamma \in \mathbb{R} \backslash S$ and $\delta \in \mathbb{R}$ are chosen so that

$$
\gamma^{2}+\delta^{2}<\alpha^{2}
$$

Then, for all $\xi \in \mathbb{R}$, the operator

$$
\begin{aligned}
A_{\gamma+i \xi, \delta}: \quad \tilde{L}_{\delta}^{2}(\mathbb{R}, \mathbb{C}) & \longrightarrow \tilde{L}_{\delta}^{2}(\mathbb{R}, \mathbb{C}), \\
W & \longmapsto L_{\gamma+i \xi} W
\end{aligned}
$$

is an isomorphism. Moreover, there exists a constant $C>0$ (depending on $\gamma$ and

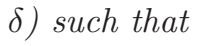

$$
\|W\|_{\tilde{L}_{\delta}^{2}(\mathbb{R}, \mathbb{C})} \leq \frac{C}{1+|\xi|^{2}}\left\|L_{\gamma+i \xi} W\right\|_{\tilde{L}_{\delta}^{2}(\mathbb{R}, \mathbb{C})},
$$

for all $W \in \tilde{W}_{\delta}^{2,2}(\mathbb{R}, \mathbb{C})$.

Proof. We assume that $\gamma \in \mathbb{R} \backslash S$ and we choose $\delta^{\prime} \in\left(-\delta_{\zeta}, 0\right]$. We argue by contradiction. Assume that $A_{\zeta, \delta^{\prime}}$ is not injective. Then, there would exist a nontrivial solution of $L_{\zeta} W=0$ which belongs to $\tilde{L}_{\delta^{\prime}}^{2}(\mathbb{R}, \mathbb{C}) \subset L^{2}(\mathbb{R}, \mathbb{C})$ and hence $W$ would be an eigenfunction of $L$. Indeed

$$
L W=\zeta^{2} W
$$

But the operator $L$ being real and selfadjoint, we find that necessarily $\zeta^{2} \in \mathbb{R}$. This implies that $\gamma \in S$, which is in contradiction with our hypothesis. Therefore, $A_{\zeta, \delta^{\prime}}$ is injective and by the previous proposition, we conclude that $A_{\zeta, \delta^{\prime}}$ is an isomorphism for any $\delta^{\prime} \in\left(-\delta_{\zeta}, \delta_{\zeta}\right)$.

We assume that $\gamma \in \mathbb{R} \backslash S$ and $\delta \in \mathbb{R}$ are chosen so that $\gamma^{2}+\delta^{2}<\alpha^{2}$. In particular $\gamma \in(-\alpha, \alpha)$ and it follows from (3.2) and Lemma 3.2 that

$$
\delta \in\left(-\delta_{\zeta}, \delta_{\zeta}\right)
$$

for all $\xi \in \mathbb{R}$, where $\zeta=\gamma+i \xi$. Therefore, we conclude that $A_{\zeta, \delta}$ is an isomorphism and this property holds independently of $\xi \in \mathbb{R}$. It remains to estimate the norm of the inverse of $A_{\zeta, \delta}$.

First observe that $\gamma$ and $\delta$ are fixed and hence $\xi$ is the only parameter allowed to vary. Clearly, the inverse of $A_{\zeta, \delta}$ is bounded uniformly in $\xi$ provided $|\xi|$ stays in 
some compact subset. Therefore, we just have to estimate the norm of the inverse of $A_{\zeta, \delta}$ when $|\xi|$ is large. To achieve this, we consider the equation

$$
L_{\zeta} W=V
$$

which we integrate against $(\cosh x)^{-2 \delta} \bar{U}$. Since we are working with complexvalued functions, we have to consider the scalar product

$$
\langle a, b\rangle=\frac{1}{2}(a \bar{b}+\bar{a} b) .
$$

After an integration by parts, we obtain

$$
\begin{array}{r}
\int_{\mathbb{R}}\left(\left|\partial_{x} W\right|^{2}-\delta \partial_{x}|W|^{2} \tanh x+\left(F^{\prime \prime}\left(u_{0}\right)-\gamma^{2}+\xi^{2}\right)|W|^{2}\right)(\cosh x)^{-2 \delta} d x \\
=\int_{\mathbb{R}}\langle W, V\rangle(\cosh x)^{-2 \delta} d x .
\end{array}
$$

A second integration by parts yields

$$
\int_{\mathbb{R}}\left(\left|\partial_{x} W\right|^{2}+\left(\xi^{2}+B\right)|W|^{2}\right)(\cosh x)^{-2 \delta} d x=\int_{\mathbb{R}}\langle W, V\rangle(\cosh x)^{-2 \delta} d x,
$$

where we have defined for short

$$
B(x):=F^{\prime \prime}\left(u_{0}(x)\right)-\gamma^{2}+\delta-\delta(2 \delta+1)(\tanh x)^{2} .
$$

Now assume that $\xi^{2}>\|B\|_{L^{\infty}}$ and use the Cauchy-Schwarz inequality to conclude that

$$
\left(\xi^{2}-\|B\|_{L^{\infty}}\right)^{2} \int_{\mathbb{R}}|W|^{2}(\cosh x)^{-2 \delta} d x \leq \int_{\mathbb{R}}|V|^{2}(\cosh x)^{-2 \delta} d x,
$$

which provides the relevant estimate for $|\xi|$ large enough. This completes the proof of the proposition.

Remark 3.1. Observe that, given $j \geq 0$, the assumption $\gamma \in \mathbb{R} \backslash S$ can be replaced by the assumption $\gamma \neq \pm \sqrt{\mu_{i}}$ for all $i \geq j+1$, but then the operator $A_{\gamma+i \xi, \delta}$ has to be restricted to the closed subspace of $\tilde{W}_{\delta}^{2,2}(\mathbb{R}, \mathbb{C})$, which consists of functions $w$ that satisfy the orthogonality condition

$$
\Pi_{i}(w)=0, \quad \text { for } \quad i=0, \ldots, j,
$$

where $\Pi_{i}$ is the projection onto the $i$-th eigenspace of the operator $L$.

\section{The Linearized operator ABOUt THE MOdel SOLUtion}

We define

$$
\mathcal{L}:=-\partial_{y}^{2}+L=-\Delta+F^{\prime \prime}\left(u_{0}\right),
$$

which is, in dimension 2, the linearized equation (2.1) about the solution $u_{0}$ which is trivially extended in the $y$ variable. We denote by $(x, y)$ the coordinates of $\mathrm{x} \in \mathbb{R}^{2}$. Given $\gamma, \delta \in \mathbb{R}$, we define the weighted space

$$
\tilde{L}_{\gamma, \delta}^{2}\left(\mathbb{R}^{2}\right):=e^{\gamma y}(\cosh x)^{\delta} L^{2}\left(\mathbb{R}^{2}\right),
$$

and similarly, we define

$$
\tilde{W}_{\gamma, \delta}^{2,2}\left(\mathbb{R}^{2}\right):=e^{\gamma y}(\cosh x)^{\delta} W^{2,2}\left(\mathbb{R}^{2}\right) .
$$

Remark 4.1. From now on all functions spaces considered are spaces of real-valued functions. 
These spaces are equipped with the natural norm induced by the weight $e^{\gamma y}(\cosh x)^{\delta}$. For example,

$$
\|w\|_{\tilde{L}_{\gamma, \delta}^{2}\left(\mathbb{R}^{2}\right)}:=\left\|e^{-\gamma y}(\cosh x)^{-\delta} w\right\|_{L^{2}\left(\mathbb{R}^{2}\right)}
$$

and so on.

4.1. Global invertibility. Given $\gamma, \delta \in \mathbb{R}$, we define the operator

$$
\begin{aligned}
\mathcal{A}_{\gamma, \delta}: \quad \tilde{L}_{\gamma, \delta}^{2}\left(\mathbb{R}^{2}\right) & \longrightarrow \tilde{L}_{\gamma, \delta}^{2}\left(\mathbb{R}^{2}\right), \\
w & \longmapsto \mathcal{L} w,
\end{aligned}
$$

which is an unbounded operator with closed graph and dense domain given by $\tilde{W}_{\gamma, \delta}^{2,2}\left(\mathbb{R}^{2}\right)$ (this fact follows easily from standard elliptic estimates). Building on the analysis of the previous section and using Fourier analysis in the $y$ variable, we prove that $\mathcal{A}_{\gamma, \delta}$ is an isomorphism (from its domain into its range) provided the weights $\gamma$ and $\delta$ are carefully chosen. More precisely, when $\gamma$ does not belong to $S$, which has been defined in (3.1) and which is the set of indicial roots of the operator $\mathcal{L}$. This is the content of the following proposition which borrows arguments from 16]:

Proposition 4.1. Assume that $\gamma \in \mathbb{R} \backslash S$ and $\delta \in \mathbb{R}$ satisfy

$$
\gamma^{2}+\delta^{2}<\alpha^{2}
$$

Then the operator $\mathcal{A}_{\gamma, \delta}$ is an isomorphism.

Proof. The proof is based on the Fourier transform in the $y$ variable. We want to solve the equation $\mathcal{L} w=v$ when $v \in \tilde{L}_{\gamma, \delta}^{2}\left(\mathbb{R}^{2}\right)$. We write

$$
w=e^{\gamma y} W \quad \text { and } \quad v=e^{\gamma y} V,
$$

so that the equation we need to solve transforms into

$$
\left(L-\gamma^{2}-2 \gamma \partial_{y}-\partial_{y}^{2}\right) W=V
$$

Now, we perform the Fourier decomposition of $W$ and $V$ in the $y$ variable. Let us denote by $\hat{W}(\cdot, x)$ and $\hat{V}(\cdot, x)$ the Fourier transforms of $W(\cdot, x)$ and $V(\cdot, x)$. Our problem then reduces to solving

$$
L_{\zeta} \hat{W}(\cdot, \xi)=\hat{V}(\cdot, \xi)
$$

which is a family of equations depending on the parameter $\zeta=\gamma+i \xi$. To solve this equation, we use the result of Proposition 3.2 and then take the inverse Fourier transform. Using Plancherel's theorem, we can write

$$
\|w\|_{\tilde{L}_{\gamma, \delta}^{2}\left(\mathbb{R}^{2}\right)}^{2}=\int_{\mathbb{R}^{2}}(\cosh x)^{-2 \delta}|W|^{2} d x d y=\int_{\mathbb{R}^{2}}(\cosh x)^{-2 \delta}|\hat{W}|^{2} d x d \xi .
$$

The result of Proposition 3.2 then implies that

$$
\|w\|_{\tilde{L}_{\gamma, \delta}^{2}\left(\mathbb{R}^{2}\right)}^{2} \leq C \int_{\mathbb{R}^{2}}(\cosh x)^{-2 \delta}|\hat{V}|^{2} d x d \xi .
$$

Using once more Plancherel's theorem, we conclude that

$$
\|w\|_{\tilde{L}_{\gamma, \delta}^{2}\left(\mathbb{R}^{2}\right)}^{2} \leq C \int_{\mathbb{R}^{2}}(\cosh x)^{-2 \delta}|V|^{2} d x d y=C\|v\|_{\tilde{L}_{\gamma, \delta}^{2}\left(\mathbb{R}^{2}\right)}^{2},
$$

and this completes the proof of the result. 
Observe that, as a by-product, we have the inequality

$$
\|w\|_{\tilde{L}_{\gamma, \delta}^{2}\left(\mathbb{R}^{2}\right)} \leq C\|\mathcal{L} w\|_{\tilde{L}_{\gamma, \delta}^{2}\left(\mathbb{R}^{2}\right)},
$$

for some constant $C>0$ only depending on $\gamma$ and $\delta$.

Remark 4.2. As in Remark 3.1, given $j \geq 0$, we can modify the hypotheses of the above proposition assuming only that $\gamma \neq \pm \sqrt{\mu_{i}}$, for all $i \geq j+1$, but then we have to consider the operator $\mathcal{A}_{\delta, \gamma}$ restricted to the closed subspace of $\tilde{W}_{\gamma, \delta}\left(\mathbb{R}^{2}\right)$ which consists of functions $w$ satisfying

$$
\Pi_{i}(w(\cdot, y))=0, \quad i=0, \ldots, j,
$$

for almost all $y \in \mathbb{R}$, where $\Pi_{i}$ is the projection onto the $i$-th eigenspace of $L$.

Let $G_{\zeta, \delta}$ denote the inverse of $A_{\zeta, \delta}$ which is provided by Proposition 3.2, It is interesting to observe that the previous proof yields a representation formula for the solution of $\mathcal{L} w=v$ which can be formally written as the integral over some contour in $\mathbb{C}$, namely

$$
w(x, y)=\frac{1}{2 \pi} \int_{\Re \zeta=\gamma} e^{\zeta y} G_{\zeta, \delta}\left(\int_{\mathbb{R}} e^{-\zeta y^{\prime}} v\left(x, y^{\prime}\right) d y^{\prime}\right) d \zeta .
$$

The estimate in Proposition 3.2 shows that the integral over $\Re \zeta=\gamma$ is well defined.

4.2. A priori estimates. In this section we explain how a priori estimates can be obtained for solutions of the equation

$$
-(\Delta-\Phi) w=v,
$$

whose potential $\Phi$ is a smooth function such that

$$
\Phi \geq \alpha^{2}-\varepsilon^{2}>0,
$$

in $\mathbb{R}^{2}$, for some $\varepsilon \in(0, \varepsilon)$. These estimates are used in the subsequent proofs. Their derivation, which relies on suitable integration by parts, is quite flexible so that it can be easily adapted in a different context.

We also assume that we are given a function $\Gamma$ which is smooth, positive and for which

$$
|\nabla \Gamma|^{2} \leq \beta^{2} \Gamma^{2}
$$

with $\beta^{2}<\alpha^{2}-\varepsilon^{2}$.

Typical examples of weight functions we consider in applications are of the form

$$
\Gamma(x, y):=e^{\delta x} e^{\gamma y}, \quad \Gamma(x, y):=e^{\delta x}(\cosh y)^{\gamma}
$$

or

$$
\Gamma(x, y):=(\cosh x)^{\delta}(\cosh y)^{\gamma},
$$

where $\gamma$ and $\delta$ are chosen so that

$$
\delta^{2}+\gamma^{2}<\alpha^{2}-\varepsilon^{2} .
$$

It is straightforward to check that these functions satisfy (4.4) with $\beta^{2}=\delta^{2}+\gamma^{2}$ (observe that $|\sinh y| \leq \cosh y$ ).

Let us now explain how a priori estimates can be obtained in this context. We assume that we are given functions $w$ and $v$ satisfying

$$
-(\Delta-\Phi) w=v
$$


in $\mathbb{R}^{2}$. We further assume that $w,|\nabla w|,\left|\nabla^{2} w\right| \in \Gamma^{-1} L^{2}\left(\mathbb{R}^{2}\right)$ and also that $v \in$ $\Gamma^{-1} L^{2}\left(\mathbb{R}^{2}\right)$. We compute

$$
-\int w \Gamma^{2}(\Delta-\Phi) w d \mathrm{x}=\int w v \Gamma^{2} d \mathbf{x}
$$

where all integrations are understood over $\mathbb{R}^{2}$. Integration by parts yields

$$
\int w^{2} \Phi \Gamma^{2} d \mathrm{x}+\int|\nabla w|^{2} \Gamma^{2} d \mathrm{x}=\int w v \Gamma^{2} d \mathrm{x}-2 \int w \Gamma \nabla w \nabla \Gamma d \mathrm{x} .
$$

Using (4.3) together with the inequality $2 a b \leq a^{2}+b^{2}$, we can estimate the last term and get

$\left(\alpha^{2}-\varepsilon^{2}\right) \int w^{2} \Gamma^{2} d \mathrm{x}+\int|\nabla w|^{2} \Gamma^{2} d \mathrm{x} \leq \int w v \Gamma^{2} d \mathrm{x}+\int|\nabla \Gamma|^{2} w^{2} d \mathrm{x}+\int|\nabla w|^{2} \Gamma^{2} d \mathrm{x}$.

Thanks to (4.4) we obtain

$$
\left(\alpha^{2}-\varepsilon^{2}-\beta^{2}\right) \int w^{2} \Gamma^{2} d \mathbf{x} \leq \int w v \Gamma^{2} d \mathbf{x},
$$

and, using the Cauchy-Schwarz inequality, we conclude that we have the a priori estimate

$$
\left(\alpha^{2}-\varepsilon^{2}-\beta^{2}\right)^{2} \int w^{2} \Gamma^{2} d \mathrm{x} \leq \int v^{2} \Gamma^{2} d \mathrm{x} .
$$

Naturally, this argument requires some care since all integrations by parts have to be justified but, in the case under study, this is standard and left to the reader.

Observe that this argument is quite flexible and can also be used when $\mathbb{R}^{2}$ is replaced by a half-space or a wedge, provided we control the function $w$ on the boundary of the domain (for example if $w$ vanishes on this boundary).

4.3. Linear Decomposition Lemma for $\mathcal{L}$. We obtain some information about the solution of the equation $\mathcal{L} w=v$ which is provided by the result of Proposition 4.1 .

The first result we prove is concerned with the asymptotic behavior of the solution $w$ in the $x$ variable when the function $v$ belongs to a function space which is smaller than the function space $\tilde{L}_{\gamma, \delta}^{2}\left(\mathbb{R}^{2}\right)$ which is used for the inversion of $\mathcal{L}$. Here is the precise statement:

Lemma 4.1. Assume that $w \in \tilde{L}_{\gamma, \delta}^{2}\left(\mathbb{R}^{2}\right)$ and $v \in \tilde{L}_{\gamma, \bar{\delta}}^{2}\left(\mathbb{R}^{2}\right)$ satisfy $\mathcal{L} w=v$ with $\bar{\delta}<\delta$. Further assume that

$$
\delta^{2}+\gamma^{2}<\alpha^{2} \quad \text { and } \quad \bar{\delta}^{2}+\gamma^{2}<\alpha^{2} .
$$

Then, $w \in \tilde{L}_{\gamma, \bar{\delta}}^{2}\left(\mathbb{R}^{2}\right)$.

Proof. To prove the lemma we choose $x_{0}>0$ and $\varepsilon>0$ such that

$$
\inf _{|x| \geq x_{0}} F^{\prime \prime}\left(u_{0}\right) \geq \alpha^{2}-\varepsilon^{2}>\delta^{2}+\gamma^{2} .
$$

Next, we define some cutoff function $\chi$ which is identically equal to 0 for $x<x_{0}$ and identically equal to 1 for $x>x_{0}+1$. We compute

$$
\mathcal{L}(\chi w)=\chi v+\left[\partial_{x}^{2}, \chi\right] w,
$$

where by definition

$$
\left[\partial_{x}^{2}, \chi\right] w:=\partial_{x}^{2}(\chi w)-\chi \partial_{x}^{2} w .
$$


We set

$$
\mathbb{R}_{x_{0}}^{2}=\left\{\mathrm{x}=(x, y) \in \mathbb{R}^{2} \quad: \quad x \geq x_{0}\right\} .
$$

Elliptic estimates imply that

$$
\|w\|_{e^{\gamma y} e^{\delta x} W^{2,2}\left(\mathbb{R}_{x_{0}}^{2}\right)} \leq C\left(\|v\|_{\tilde{L}_{\gamma, \bar{\delta}}^{2}\left(\mathbb{R}^{2}\right)}+\|w\|_{\tilde{L}_{\gamma, \delta}^{2}\left(\mathbb{R}^{2}\right)}\right),
$$

and hence, we conclude that

$$
\left\|\chi v+\left[\partial_{x}^{2}, \chi\right] w\right\|_{e^{\gamma y} e^{\bar{\delta} x} L^{2}\left(\mathbb{R}_{x_{0}}^{2}\right)} \leq C\left(\|v\|_{\tilde{L}_{\gamma, \bar{\delta}}^{2}\left(\mathbb{R}^{2}\right)}+\|w\|_{\tilde{L}_{\gamma, \delta}^{2}\left(\mathbb{R}^{2}\right)}\right)
$$

for some constant $C>0$ (depending on $x_{0}$ ).

We claim that there exists a function $\bar{w} \in e^{\gamma y} e^{\bar{\delta} x} L^{2}\left(\mathbb{R}_{x_{0}}^{2}, \mathbb{R}\right)$ such that

$$
\mathcal{L} \bar{w}=\mathcal{L}(\chi w),
$$

in $\mathbb{R}_{x_{0}}^{2}$ and $\bar{w}=0$ on $\partial \mathbb{R}_{x_{0}}^{2}$. To prove the claim, we first solve the equation in $\left[x_{0}, x_{1}\right] \times\left[-y_{0}, y_{0}\right]$ under homogeneous Dirichlet boundary conditions, denote this solution by $\bar{w}_{x_{1}, y_{0}}$, and then let $x_{1}$ and $y_{0}$ tend to infinity. To pass to the limit, we use the a priori estimate of the previous section to get

$$
\left\|\bar{w}_{x_{1}, y_{0}}\right\|_{e^{\gamma y} e^{\bar{\delta} x} L^{2}\left(\left[x_{0}, x_{1}\right] \times\left[-y_{0}, y_{0}\right]\right)} \leq C\|\mathcal{L}(\chi w)\|_{e^{\gamma y} e^{\bar{\delta} x} L^{2}\left(\mathbb{R}_{x_{0}}^{2}\right)},
$$

where the constant $C>0$ depends neither on $x_{1}>x_{0}+1$ nor on $y_{0}>1$. An elliptic estimate together with this a priori estimate allows us to pass to the limit as $x_{1}$ and $y_{0}$ tend to infinity. This completes the proof of the claim.

Finally, we use once more the a priori estimate to show that $\chi w=\bar{w}$ in $\mathbb{R}_{x_{0}}^{2}$. Indeed, by construction, $\mathcal{L}(\bar{w}-\chi w)=0$ in $\mathbb{R}_{x_{0}}^{2}$ vanishes on the boundary of this set and $\bar{w}-\chi w \in e^{\gamma y} e^{\delta x} L^{2}\left(\mathbb{R}_{x_{0}}^{2}\right)$. The a priori estimate of the previous section implies that

$$
\|\bar{w}-\chi w\|_{e^{\gamma y} e^{\delta x} L^{2}\left(\mathbb{R}_{x_{0}}^{2}\right)} \leq C\|\mathcal{L}(\bar{w}-\chi w)\|_{e^{\gamma y} e^{\delta x} L^{2}\left(\mathbb{R}_{x_{0}}^{2}\right)}=0 .
$$

Therefore, we conclude that $w \in e^{\gamma y} e^{\bar{\delta} x} L^{2}\left(\mathbb{R}_{x_{0}}^{2}\right)$. A similar argument applies in

$$
\mathbb{R}_{-x_{0}}^{2}=\left\{\mathrm{x}=(x, y) \in \mathbb{R}^{2} \quad: \quad x \leq-x_{0}\right\},
$$

thus ending the proof.

We define the function space

$$
\mathbb{L}_{\gamma, \delta}^{2}\left(\mathbb{R}^{2}\right):=(\cosh y)^{\gamma}(\cosh x)^{\delta} L^{2}\left(\mathbb{R}^{2}\right) .
$$

Observe that when $\gamma \geq 0$,

$$
\mathbb{L}_{-\gamma, \delta}^{2}\left(\mathbb{R}^{2}\right) \subset \tilde{L}_{\gamma, \delta}^{2}\left(\mathbb{R}^{2}\right) .
$$

We are now concerned with the behavior of the solution $w$ in the $y$ variable when the function $v$ belongs to $\mathbb{L}_{-\gamma, \delta}^{2}\left(\mathbb{R}^{2}\right)$ which is a smaller space than the function space $\tilde{L}_{\gamma, \delta}^{2}\left(\mathbb{R}^{2}\right)$ which is used for the inversion of $\mathcal{L}$. To state the relevant result we let $\chi$ be a cutoff function which is identically 0 in $(-\infty,-1)$ and identically 1 in $(1,+\infty)$. With this notation the following holds:

Lemma 4.2 (Linear Decomposition Lemma). Let $\mu_{j}<\mu_{j+1}$ be two consecutive eigenvalues of the operator $L:=-\partial_{x}^{2}+F^{\prime \prime}\left(u_{0}\right)$. We assume that

$$
\sqrt{\mu}_{j}<\gamma<\sqrt{\mu_{j+1}}
$$

and also that

$$
\gamma^{2}+\delta^{2}<\alpha^{2}
$$


We further assume that $w \in \tilde{L}_{\gamma, \delta}^{2}\left(\mathbb{R}^{2}\right)$ and

$$
\mathcal{L} w=v \in \mathbb{L}_{-\gamma, \delta}^{2}\left(\mathbb{R}^{2}\right)
$$

Then, for $i=0, \ldots, j$, there exist $w_{i}, \bar{w}_{i}$ in the $i$-th eigenspace of $L$ and $\bar{w} \in$ $\mathbb{L}_{-\gamma, \delta}^{2}\left(\mathbb{R}^{2}\right)$ such that

$w(x, y)=\bar{w}(x, y)+\chi(y)\left(w_{0}(x)+y \bar{w}_{0}(x)\right)+\sum_{i=1}^{j} \chi(y)\left(w_{i}(x) e^{\sqrt{\mu}_{i} y}+\bar{w}_{i}(x) e^{-\sqrt{\mu}_{i} y}\right)$

(we agree that the last sum is not present when $j=0$ ). Moreover,

$$
\sum_{i=0}^{j}\left(\left\|w_{i}\right\|_{L^{2}(\mathbb{R})}+\left\|\bar{w}_{i}\right\|_{L^{2}(\mathbb{R})}\right)+\|\bar{w}\|_{\mathbb{L}_{-\gamma, \delta}^{2}\left(\mathbb{R}^{2}\right)} \leq C\|v\|_{\mathbb{L}_{-\gamma, \delta}^{2}\left(\mathbb{R}^{2}\right)},
$$

for some constant $C>0$ only depending on $\gamma$ and $\delta$.

Proof. Recall that $\Pi_{i}$ is the $L^{2}$-orthogonal projection over the $i$-th eigenspace of $L$. We decompose

$$
w=w^{\perp}+\sum_{i=0}^{j} w_{i}^{\|} \quad \text { and } \quad v=v^{\perp}+\sum_{i=0}^{j} v_{i}^{\|}
$$

where, for all $i=0, \ldots, j$,

$$
\Pi_{i}\left(w^{\perp}\right)=0, \quad \Pi_{i}\left(v^{\perp}\right)=0,
$$

and

$$
w_{i}^{\|}=\Pi_{i}(w), \quad v_{i}^{\|}=\Pi_{i}(v),
$$

for almost every $y \in \mathbb{R}$.

Recall that, mutatis mutandis, the result of Proposition 4.1 holds when we restrict our attention to the space of functions $w$ satisfying

$$
\Pi_{i}(w)=0 \quad \text { for almost every } \quad y \in \mathbb{R} \quad \text { and } \quad i=0, \ldots, j
$$

(see Remark 4.2), the only difference being that the restriction on $\gamma$ is now given by $\gamma \neq \pm \sqrt{\mu}_{i+1}$ for all $i \geq j$.

Thanks to the analysis of the previous section, we can write

$$
w^{\perp}(x, y)=\frac{1}{2 \pi} \int_{\Re \zeta=\gamma} e^{-\zeta y} G_{\zeta, \delta}\left(\int_{\mathbb{R}} e^{\zeta y^{\prime}} v^{\perp}\left(x, y^{\prime}\right) d y^{\prime}\right) d \zeta .
$$

Let us assume that, in addition, $v^{\perp}$ has compact support (the general result follows by density). The integrand depends analytically on $\zeta$ in the set

$$
\left\{\zeta \in \mathbb{C}: \Re \zeta \in\left(-\sqrt{\mu_{j+1}}, \sqrt{\mu_{j+1}}\right)\right\},
$$

since we are working under the assumption that all functions do satisfy (4.7). Therefore, the Cauchy formula implies that

$$
\frac{1}{2 \pi} \int_{\Re \zeta=\gamma} e^{-\zeta y} G_{\zeta, \delta}\left(\int_{\mathbb{R}} e^{\zeta y^{\prime}} v^{\perp}\left(x, y^{\prime}\right) d y^{\prime}\right) d \zeta
$$


does not depend on $\gamma \in\left(-\sqrt{\mu_{j+1}}, \sqrt{\mu_{j+1}}\right)$. Consequently, we have

$$
w^{\perp}(x, y)=\frac{1}{2 \pi} \int_{\Re \zeta=-\gamma} e^{-\zeta y} G_{\zeta, \delta}\left(\int_{\mathbb{R}} e^{\zeta y^{\prime}} v^{\perp}\left(x, y^{\prime}\right) d y^{\prime}\right) d \zeta,
$$

which implies that

$$
w^{\perp} \in \tilde{L}_{-\gamma, \delta}^{2}\left(\mathbb{R}^{2}\right) \cap \tilde{L}_{\gamma, \delta}^{2}\left(\mathbb{R}^{2}\right)=\mathbb{L}_{-\gamma, \delta}^{2}\left(\mathbb{R}^{2}\right) .
$$

It remains to prove the result for $w_{i}^{\|}$. Observe that, this time, the problem reduces to solving an ordinary differential equation

$$
\left(-\partial_{y}^{2}+\mu_{i}\right) w_{i}^{\|}=v_{i}^{\|} .
$$

It is easy to check that the solution is explicitly given by

$$
w_{i}^{\|}(x, y)= \begin{cases}-\int_{-\infty}^{y} \int_{-\infty}^{y^{\prime}} v_{i}^{\|}\left(x, y^{\prime \prime}\right) d y^{\prime \prime} d y^{\prime}, & \text { when } i=0, \\ \frac{1}{2 \sqrt{\mu_{i}}} \int_{-\infty}^{y}\left(e^{\sqrt{\mu_{i}}\left(y^{\prime}-y\right)}-e^{\sqrt{\mu_{i}}\left(y-y^{\prime}\right)}\right) v_{i}^{\|}\left(x, y^{\prime}\right) d y^{\prime}, & \text { when } 0<i \leq j .\end{cases}
$$

Moreover, when $0<i \leq j$, the function $w_{i}^{\|}$can be decomposed as

$$
w_{i}^{\|}=\bar{w}_{i}^{\|}+\chi(y)\left(w_{i} e^{\sqrt{\mu_{i}} y}+\bar{w}_{i} e^{-\sqrt{\mu_{i}} y}\right),
$$

where $\bar{w}_{i}^{\|} \in \mathbb{L}_{-\gamma, \delta}^{2}\left(\mathbb{R}^{2}, \mathbb{R}\right)$ and $w_{i}, \bar{w}_{i}$ belong to the $i$-th eigenspace of $L$. A similar decomposition can be done when $i=0$. The estimate in the statement of the result follows at once from the proof and is left to the reader.

Since we will only use this result in the case where $\gamma \in\left(0, \sqrt{\mu_{1}}\right)$, namely when $j=0$, let us examine this case more closely, giving at the same time another consequence of the Linear Decomposition Lemma. Keeping in mind the applications we are interested in, we fix $\gamma \in\left(0, \sqrt{\mu}_{1}\right)$ and $\delta \in \mathbb{R}$ such that $\delta^{2}+\gamma^{2}<\alpha^{2}$. It is useful to introduce the operator $\mathbb{A}_{-\gamma, \delta}$, defined by

$$
\mathbb{A}_{-\gamma, \delta}: \quad \mathbb{L}_{-\gamma, \delta}^{2}\left(\mathbb{R}^{2}\right) \oplus \mathbb{D} \quad \longrightarrow \quad \mathbb{L}_{-\gamma, \delta}^{2}\left(\mathbb{R}^{2}\right),
$$

$$
w \quad \longmapsto \quad \mathcal{L} w,
$$

where $\mathbb{D}$ is the finite-dimensional space defined by

$$
\mathbb{D}:=\operatorname{Span}\left\{\begin{array}{rlrl}
(x, y) & \longmapsto \chi(y) \partial_{x} u_{0}(x), & (x, y) \longmapsto(1-\chi(y)) \partial_{x} u_{0}(x), \\
(x, y) \longmapsto \chi(y) y \partial_{x} u_{0}(x), & (x, y) \longmapsto(1-\chi(y)) y \partial_{x} u_{0}(x)
\end{array}\right\} .
$$

Usually, $\mathbb{D}$ is referred to as the deficiency space.

We now prove that the operator $\mathbb{A}_{-\gamma, \delta}$ is surjective and that it has a twodimensional kernel. Notice that the deficiency space is 4-dimensional and that it can be decomposed into

$$
\mathbb{D}=\mathbb{K} \oplus \mathbb{E},
$$

where

$$
\mathbb{K}=\operatorname{Span}\left\{(x, y) \longmapsto \partial_{x} u_{0}(x), \quad(x, y) \longmapsto y \partial_{x} u_{0}(x)\right\}
$$

and

$$
\mathbb{E}=\operatorname{Span}\left\{(x, y) \longmapsto \chi(y) \partial_{x} u_{0}(x), \quad(x, y) \longmapsto \chi(y) y \partial_{x} u_{0}(x)\right\}
$$


We claim that the Linear Decomposition Lemma implies that the unbounded operator

$$
\begin{aligned}
& \overline{\mathbb{A}}_{-\gamma, \delta}: \quad \mathbb{L}_{-\gamma, \delta}^{2}\left(\mathbb{R}^{2}\right) \oplus \mathbb{E} \quad \longrightarrow \quad \mathbb{L}_{-\gamma, \delta}^{2}\left(\mathbb{R}^{2}\right), \\
& w \quad \longmapsto \quad \mathcal{L} w
\end{aligned}
$$

is an isomorphism. Indeed, if $v \in \mathbb{L}_{-\gamma, \delta}^{2}\left(\mathbb{R}^{2}\right)$, Proposition 4.1 provides the existence of a solution $w$ of $\mathcal{L} w=v$ with $w \in \tilde{L}_{\gamma, \delta}^{2}\left(\mathbb{R}^{2}\right)$. The Linear Decomposition Lemma then implies that $w \in \mathbb{L}_{-\gamma, \delta}^{2}\left(\mathbb{R}^{2}\right) \oplus \mathbb{E}$ and this proves that the operator $\overline{\mathbb{A}}_{-\gamma, \delta}$ is surjective. Clearly $\mathbb{K}$ contains the kernel of $\mathbb{A}_{-\gamma, \delta}$, and it suffices to show that this set is precisely equal to the kernel of $\mathbb{A}_{-\gamma, \delta}$. To proceed, we assume that $w \in \mathbb{L}_{-\gamma, \delta}^{2}\left(\mathbb{R}^{2}\right) \oplus \mathbb{E}$ satisfies $\mathcal{L} w=0$. Then, $w \in \tilde{L}_{\gamma, \delta}^{2}\left(\mathbb{R}^{2}\right)$ and Proposition 4.1 implies that $w \equiv 0$, which completes the proof of the claim. As a by-product, we have shown that the operator $\mathbb{A}_{-\gamma, \delta}$ is surjective and has a 2-dimensional kernel equal to $\mathbb{K}$.

All the above results can be extended, with obvious modifications, to handle the case where $\gamma$ is chosen between two consecutive indicial roots of $\mathcal{L}$, namely when $\sqrt{\mu_{j}}<\gamma<\sqrt{\mu_{j+1}}$.

\section{The linearized operator about an Element of $\mathcal{M}_{2 k}$}

In this section, we first explain how the previous results can be put together to obtain similar results for the linearized operator about the function $u_{\lambda}$ which was defined in (2.6), where $\lambda:=\left(\lambda_{1}, \ldots, \lambda_{2 k}\right) \in \Lambda_{\text {ord }}^{2 k}$. We then show that these results extend to the operator linearized about any element of $\mathcal{M}_{2 k}$.

To begin with, we assume that $\lambda \in \Lambda_{\text {ord }}^{2 k}$ is fixed and we denote by $\mathfrak{L}_{\lambda}$ the linearized operator about $u_{\lambda}$, namely

$$
\mathfrak{L}_{\lambda}=-\Delta+F^{\prime \prime}\left(u_{\lambda}\right),
$$

and we derive an a priori estimate for this operator in the space $L_{\gamma, \delta}^{2}\left(\mathbb{R}^{2}\right)$. Recall that this space is defined in (2.11). In this analysis, we also need to consider the weighted space $W_{\gamma, \delta}^{2,2}\left(\mathbb{R}^{2}\right)$, which was defined in (2.12). The following result follows from the previous analysis together with a careful use of cutoff functions. It shows the crucial role played by $S$, the set of indicial roots of $\mathcal{L}$, in our analysis:

Proposition 5.1. Assume that $\gamma \in \mathbb{R} \backslash S$ and

$$
\delta^{2}+\gamma^{2}<\alpha^{2} \text {. }
$$

Then, there exist $\bar{R} \geq R$ and $C>0$ such that, for all $w, v \in L_{\gamma, \delta}^{2}\left(\mathbb{R}^{2}\right)$ satisfying

$$
\mathfrak{L}_{\lambda} w=v,
$$

we have

$$
\|w\|_{L_{\gamma, \delta}^{2}\left(\mathbb{R}^{2}\right)} \leq C\left(\|v\|_{L_{\gamma, \delta}^{2}\left(\mathbb{R}^{2}\right)}+\|w\|_{L^{2}\left(B_{\bar{R}}\right)}\right) .
$$

Proof. First of all, observe that elliptic estimates immediately imply that

$$
\|w\|_{W_{\gamma, \delta}^{2,2}\left(\mathbb{R}^{2}\right)} \leq C\left(\|v\|_{L_{\gamma, \delta}^{2}\left(\mathbb{R}^{2}\right)}+\|w\|_{L_{\gamma, \delta}^{2}\left(\mathbb{R}^{2}\right)}\right) .
$$

To proceed, we keep the notation of \$2.1. In particular, the oriented half-lines $\lambda_{j}^{+}$, which correspond to the asymptotes of the nodal curves of $u_{\lambda}$, are parameterized by

$$
(0, \infty) \ni s \longmapsto \mathrm{x}_{j}+s \mathrm{e}_{j} \in \mathbb{R}^{2} .
$$


For notational purposes, it is convenient to extend the sequence of oriented halflines $\lambda_{1}^{+}, \ldots, \lambda_{2 k}^{+}$periodically by setting $\lambda_{j+2 k}^{+}:=\lambda_{j}^{+}, \mathrm{x}_{j+2 k}:=\mathrm{x}_{j}$ and $\mathrm{e}_{j+2 k}=\mathrm{e}_{j}$ for any $j \in \mathbb{Z}$.

We denote by $\lambda_{j+\frac{1}{2}}^{+}$the oriented half-line bisecting the lines containing $\lambda_{j}^{+}$and $\lambda_{j+1}^{+}$parameterized by

$$
s \in(0, \infty) \longmapsto \mathrm{x}_{j+\frac{1}{2}}+s \mathrm{e}_{j+\frac{1}{2}} \in \mathbb{R}^{2},
$$

where

$$
\mathrm{e}_{j+\frac{1}{2}}:=\frac{\mathrm{e}_{\mathrm{j}}+\mathrm{e}_{\mathrm{j}+1}}{2}
$$

and $\mathrm{x}_{j+\frac{1}{2}} \in \partial B_{R}$. This point is contained in the angular arc of $\partial B_{R}$ (assuming its positive orientation) starting at $\mathrm{x}_{\mathrm{j}}$ and ending at $\mathrm{x}_{\mathrm{j}+1}$.

Recall that we have defined $\Omega_{j}$ in (2.5) which is a connected component of $\mathbb{R}^{2}$ which contains $\lambda_{j}^{+}$. Similarly, we define the angular sector $\Omega_{j+\frac{1}{2}}$ which is the connected component of $\mathbb{R}^{2} \backslash\left(\lambda_{j}^{+} \cup \lambda_{j+1}^{+} \cup \partial B_{R}\right)$ containing $\lambda_{j+\frac{1}{2}}^{+}$.

We need to introduce two more angular sectors

$$
\Omega_{j+1 / 2}^{\prime \prime} \subset \Omega_{j+1 / 2}^{\prime} \subset \Omega_{j+1 / 2}
$$

which are both included in the connected component of $\mathbb{R}^{2} \backslash\left(\lambda_{j}^{+} \cup \lambda_{j+1}^{+} \cup \partial B_{R}\right)$ which contains $\lambda_{j+\frac{1}{2}}^{+}$. The sector $\Omega_{j+1 / 2}^{\prime}$ is limited by $\partial B_{R}$ and the two half-lines $\lambda_{j+1 / 4}^{+}$and $\lambda_{j+3 / 4}^{+}$respectively bisecting $\lambda_{j}^{+}$and $\lambda_{j+1 / 2}^{+}$for the former and $\lambda_{j+1 / 2}^{+}$ and $\lambda_{j+1}^{+}$for the latter. The sector $\Omega_{j+1 / 2}^{\prime \prime}$ is limited by $\partial B_{R}$ and the two halflines $\lambda_{j+3 / 8}^{+}$and $\lambda_{j+5 / 8}^{+}$respectively bisecting $\lambda_{j+1 / 4}^{+}$and $\lambda_{j+1 / 2}^{+}$for the former and $\lambda_{j+1 / 2}^{+}$and $\lambda_{j+3 / 4}^{+}$for the latter.

Finally, for all $j=1, \ldots, 2 k$, we define the cutoff function $\mathbb{I}_{j+\frac{1}{2}}$ such that:

(i) $\mathbb{I}_{j+\frac{1}{2}} \equiv 0$ in the set $\mathbb{R}^{2} \backslash \Omega_{j+1 / 2}^{\prime}$,

(ii) $\mathbb{I}_{j+\frac{1}{2}} \equiv 1$ in the sector $\Omega_{j+1 / 2}^{\prime \prime}$,

(iii) $\left|\nabla^{\ell} \mathbb{I}_{j+\frac{1}{2}}(\mathrm{x})\right| \leq \mathrm{C}(1+|\mathrm{x}|)^{-\ell}$, for $\ell=0,1,2$.

Observe that (i)-(iii) imply that the support of $\mathbb{I}_{j+\frac{1}{2}}$ is an expanding, wedge-like set centered around $\lambda_{j+\frac{1}{2}}^{+}$and which is contained in $\Omega_{j+1 / 2}^{\prime}$.

We write

$$
\gamma \mathbf{e}_{j}+\delta \mathbf{e}_{j}^{\perp}=\tilde{\gamma}_{j+\frac{1}{2}} \mathbf{e}_{j+\frac{1}{2}}+\tilde{\delta}_{j+\frac{1}{2}} \mathrm{e}_{j+\frac{1}{2}}^{\perp},
$$

where $\tilde{\gamma}_{j+\frac{1}{2}}^{2}+\tilde{\delta}_{j+\frac{1}{2}}^{2}=\gamma^{2}+\delta^{2}$. Elementary geometry implies that

$$
\gamma \mathbf{e}_{j+1}-\delta \mathbf{e}_{j+1}^{\perp}=\tilde{\gamma}_{j+\frac{1}{2}} \mathbf{e}_{j+\frac{1}{2}}-\tilde{\delta}_{j+\frac{1}{2}} \mathrm{e}_{j+\frac{1}{2}}^{\perp}
$$

since $\mathbf{e}_{j+1}$ and $\mathbf{e}_{j}$ are symmetric with respect to the reflection leaving $\mathbf{e}_{j+\frac{1}{2}}$ fixed. 
We set

$$
\Gamma_{\tilde{\gamma}_{j+\frac{1}{2}}, \tilde{\delta}_{j+\frac{1}{2}}}(\mathrm{x}):=e^{\tilde{\gamma}_{j+\frac{1}{2}}\left(\mathrm{x} \cdot \mathrm{e}_{j+\frac{1}{2}}\right)}\left(\cosh \left(\mathrm{x} \cdot \mathrm{e}_{j+\frac{1}{2}}^{\perp}\right)\right)^{\tilde{\delta}_{j+\frac{1}{2}}} .
$$

Observe that the weights $\Gamma_{\gamma, \delta}$ and $\Gamma_{\tilde{\gamma}_{j+\frac{1}{2}}, \tilde{\delta}_{j+\frac{1}{2}}}$ are equivalent in the sector $\Omega_{j+\frac{1}{2}}$. Namely, there exists $C>1$ such that

$$
\frac{1}{C} \Gamma_{\gamma, \delta} \leq \Gamma_{\tilde{\gamma}_{j+\frac{1}{2}}, \tilde{\delta}_{j+\frac{1}{2}}} \leq C \Gamma_{\gamma, \delta}
$$

in $\Omega_{j+\frac{1}{2}}$

Now, if $\mathfrak{L}_{\lambda} w=v$, then

$$
\mathfrak{L}_{\lambda}\left(\mathbb{I}_{j+\frac{1}{2}} w\right)=\mathbb{I}_{j+\frac{1}{2}} v+\left[\mathfrak{L}_{\lambda}, \mathbb{I}_{j+\frac{1}{2}}\right] w,
$$

where, as usual,

$$
\left[\mathfrak{L}_{\lambda}, \mathbb{I}_{j+\frac{1}{2}}\right] w:=\mathfrak{L}_{\lambda}\left(\mathbb{I}_{j+\frac{1}{2}} w\right)-\mathbb{I}_{j+\frac{1}{2}}\left(\mathfrak{L}_{\lambda} w\right) .
$$

Given $\varepsilon>0$ small enough, there exists $\bar{R} \geq R>0$ such that

$$
F^{\prime \prime}\left(u_{\lambda}\right) \geq \alpha^{2}-\varepsilon^{2},
$$

in the support of $\mathbb{I}_{j+\frac{1}{2}}$ and away from $B_{\bar{R}}$. Making use of an argument similar to the one we used to obtain (4.5), we get

$$
\begin{aligned}
\left(\alpha^{2}-\varepsilon^{2}-\tilde{\beta}_{j+1 / 2}^{2}\right)^{2} & \int_{\mathbb{R}^{2} \backslash B_{\bar{R}}} \Gamma_{-\tilde{\gamma}_{j+\frac{1}{2}},-\tilde{\delta}_{j+\frac{1}{2}}}^{2}\left|\mathbb{I}_{j+\frac{1}{2}} w\right|^{2} d \mathrm{x} \\
\leq & C\left(\int_{\mathbb{R}^{2} \backslash B_{\bar{R}}} \Gamma_{-\tilde{\gamma}_{j+\frac{1}{2}},-\tilde{\delta}_{j+\frac{1}{2}}}^{2}\left|\mathbb{I}_{j+\frac{1}{2}} v\right|^{2} d \mathrm{x}\right. \\
& \quad+\int_{\mathbb{R}^{2} \backslash B_{\bar{R}}} \Gamma_{-\tilde{\gamma}_{j+\frac{1}{2}},-\tilde{\delta}_{j+\frac{1}{2}}}^{2}\left|\left[\mathfrak{L}_{\lambda}, \mathbb{I}_{j+\frac{1}{2}}\right] w\right|^{2} d \mathrm{x} \\
& \left.+\int_{\partial B_{\bar{R}}} \Gamma_{-\tilde{\gamma}_{j+\frac{1}{2}},-\tilde{\delta}_{j+\frac{1}{2}}}^{2}\left|\partial_{r}\left(\mathbb{I}_{j+\frac{1}{2}} w\right)\right| \mathbb{I}_{j+\frac{1}{2}} w d \mathrm{x}\right),
\end{aligned}
$$

where $\tilde{\beta}_{j+1 / 2}^{2}:=\tilde{\delta}_{j+\frac{1}{2}}^{2}+\tilde{\gamma}_{j+\frac{1}{2}}^{2}$. Since $\Gamma_{\gamma, \delta}$ and $\Gamma_{\tilde{\gamma}_{j+\frac{1}{2}}, \tilde{\delta}_{j+\frac{1}{2}}}$ are equivalent in $\Omega_{j+\frac{1}{2}}$, the first term on the right-hand side can be estimated by a constant (independent of $\bar{R}$ ) times $\|v\|_{L_{\gamma, \delta}^{2}\left(\mathbb{R}^{2}\right)}$. Using the fact that $\mathbb{I}_{j+\frac{1}{2}}$ satisfies property (iii), we conclude that the second term on the right-hand side is bounded by a constant (independent of $\bar{R})$ times $\bar{R}^{-1}\|w\|_{L_{\gamma, \delta}^{2}\left(\mathbb{R}^{2}\right)}$. Finally, using standard elliptic estimates together with a trace embedding, one can check that the third term on the right-hand side can be estimated by a constant (depending on $\bar{R}$ ) times $\|w\|_{L^{2}\left(B_{\bar{R}+1}\right)}$. Using once more the fact that the weights $\Gamma_{\gamma, \delta}$ and $\Gamma_{\tilde{\gamma}_{j+\frac{1}{2}}, \tilde{\delta}_{j+\frac{1}{2}}}$ are equivalent in the sector we are working in, we conclude that

$$
\left\|\mathbb{I}_{j+\frac{1}{2}} w\right\|_{L_{\gamma, \delta}^{2}\left(\mathbb{R}^{2}\right)} \leq C\|v\|_{L_{\gamma, \delta}^{2}\left(\mathbb{R}^{2}\right)}+C_{\bar{R}}\|w\|_{L^{2}\left(B_{\bar{R}+1}\right)}+C \bar{R}^{-1}\|w\|_{L_{\gamma, \delta}^{2}\left(\mathbb{R}^{2}\right)},
$$

where $C_{\bar{R}}>0$ depends on $\bar{R}$ but $C>0$ does not.

Given $j=1, \ldots, 2 k$, we now estimate the function $\mathbb{I}_{j} w$. Applying some rigid motion and changing the sign of $u_{\lambda}$ if this is necessary, we can assume that $\lambda_{j}$ is the $y$-axis and that $u_{\lambda}=u_{0}$ near $\lambda_{j}^{+}$. In particular, $\mathfrak{L}_{\lambda}$ coincides with the operator $\mathcal{L}$ defined in $\S 4$ close to $\lambda_{j}$. The function $\mathbb{I}_{j} w$ satisfies

$$
\mathcal{L}\left(\mathbb{I}_{j} w\right)=\mathbb{I}_{j} v+\left[\mathfrak{L}_{\lambda}, \mathbb{I}_{j}\right] w+\left(\mathcal{L}-\mathfrak{L}_{\lambda}\right)\left(\mathbb{I}_{j} w\right) .
$$


We can then apply the result of Proposition 4.1 and in particular we can make use of (4.1). To estimate the second term in (5.5), we use the definition of the $\mathbb{I}_{j}$ (see section 2.1), together with elliptic estimates to get

$$
\left\|\left[\mathfrak{L}_{\lambda}, \mathbb{I}_{j}\right] w\right\|_{L_{\gamma, \delta}^{2}\left(\mathbb{R}^{2}\right)} \leq C\left\|\mathbb{I}_{j+\frac{1}{2}} w\right\|_{L_{\gamma, \delta}^{2}\left(\mathbb{R}^{2}\right)}+C_{\bar{R}}\|w\|_{L^{2}\left(B_{\bar{R}+1}\right)},
$$

where, as usual, $C_{\bar{R}}>0$ depends on $\bar{R}$ while $C>0$ does not.

Using the definition of $u_{\lambda}$ given in (2.6), one can check that there exists $\gamma_{0}>0$ such that

$$
\left|F^{\prime \prime}\left(u_{\lambda}\right)-F^{\prime \prime}\left(u_{0}\right)\right| \leq C\left|u_{\lambda}-u_{0}\right| \leq C e^{-\gamma_{0} \bar{R}}
$$

in the set where $\mathbb{I}_{j}>0$ and $|\mathrm{x}|>\bar{R}$. Therefore, we get

$$
\left\|\left(\mathcal{L}-\mathfrak{L}_{\lambda}\right)\left(\mathbb{I}_{j} w\right)\right\|_{L_{\gamma, \delta}^{2}\left(\mathbb{R}^{2}\right)} \leq C_{\bar{R}}\|w\|_{L^{2}\left(B_{\bar{R}}+1\right)}+C e^{-\gamma_{0} \bar{R}}\|w\|_{L_{\gamma, \delta}^{2}\left(\mathbb{R}^{2}\right)} .
$$

Collecting these estimates, we conclude that

$$
\begin{aligned}
\left\|\mathbb{I}_{j} w\right\|_{L_{\gamma, \delta}^{2}\left(\mathbb{R}^{2}\right)} \leq & C\|v\|_{L_{\gamma, \delta}^{2}\left(\mathbb{R}^{2}\right)}+C_{\bar{R}}\|w\|_{L^{2}\left(B_{\bar{R}+1}\right)} \\
& +C\left\|\mathbb{I}_{j+\frac{1}{2}} w\right\|_{L_{\gamma, \delta}^{2}\left(\mathbb{R}^{2}\right)}+C e^{-\gamma_{0} \bar{R}}\|w\|_{L_{\gamma, \delta}^{2}\left(\mathbb{R}^{2}\right)} .
\end{aligned}
$$

When $j=0$, the corresponding estimate for $\mathbb{I}_{0} w$ is straightforward and left to the reader. In any case, (5.4) and (5.6) imply that

$$
\|w\|_{L_{\gamma, \delta}^{2}\left(\mathbb{R}^{2}\right)} \leq C\|v\|_{L_{\gamma, \delta}^{2}\left(\mathbb{R}^{2}\right)}+C_{\bar{R}}\|w\|_{L^{2}\left(B_{\bar{R}}+1\right)}+C\left(e^{-\gamma_{0} \bar{R}}+\bar{R}^{-1}\right)\|w\|_{L_{\gamma, \delta}^{2}\left(\mathbb{R}^{2}\right)},
$$

where $C_{\bar{R}}>0$ depends on $\bar{R}$ while $C>0$ does not. The proof of the main estimate then follows by taking $\bar{R}$ large enough.

As a corollary of the previous proposition, we obtain some a priori estimate for solutions of $\mathfrak{L} w=v$, for any $u \in \mathcal{M}_{2 k}$ :

Corollary 5.1. Assume that $u \in \mathcal{M}_{2 k}$ and let

$$
\mathfrak{L}:=-\Delta+F^{\prime \prime}(u)
$$

be the linearized operator about $u$. Let $\gamma, \delta$ be chosen so that they satisfy the hypothesis of Proposition 5.1. Then, there exist $\bar{R} \geq R$ and $C>0$ such that, for all $w, v \in L_{\gamma, \delta}^{2}\left(\mathbb{R}^{2}\right)$, satisfying

$$
\mathfrak{L} w=v,
$$

the following inequality holds:

$$
\|w\|_{L_{\gamma, \delta}^{2}\left(\mathbb{R}^{2}\right)} \leq C\left(\|v\|_{L_{\gamma, \delta}^{2}\left(\mathbb{R}^{2}\right)}+\|w\|_{L^{2}\left(B_{\bar{R}}\right)}\right) .
$$

Proof. The proof is a simple consequence of a classical perturbation argument. Indeed, by definition, if $u \in \mathcal{M}_{2 k}$, there exists $\lambda \in \Lambda$ such that $u-u_{\lambda} \in W^{2,2}\left(\mathbb{R}^{2}\right)$. Thanks to Sobolev embedding, for any $\varepsilon$, there exists $\bar{R}_{\varepsilon} \geq \bar{R}$ such that

$$
\left\|F^{\prime \prime}(u)-F^{\prime \prime}\left(u_{\lambda}\right)\right\|_{L^{\infty}\left(\mathbb{R}^{2} \backslash B_{R_{\varepsilon}}\right)} \leq \varepsilon .
$$

Hence

$$
\left\|\left(F^{\prime \prime}(u)-F^{\prime \prime}\left(u_{\lambda}\right)\right) w\right\|_{L_{\gamma, \delta}^{2}\left(\mathbb{R}^{2}\right)} \leq C_{\varepsilon}\|w\|_{L^{2}\left(B_{\bar{R}_{\varepsilon}}\right)}+\varepsilon\|w\|_{L_{\gamma, \delta}^{2}\left(\mathbb{R}^{2}\right)},
$$

for some constant $C_{\varepsilon}>0$ (depending on $\varepsilon$ ). Since $\mathfrak{L}_{\lambda} w=v+\left(F^{\prime \prime}\left(u_{\lambda}\right)-F^{\prime \prime}(u)\right) w$, it follows from Proposition 5.1 that

$$
\|w\|_{L_{\gamma, \delta}^{2}\left(\mathbb{R}^{2}\right)} \leq C\|v\|_{L_{\gamma, \delta}^{2}\left(\mathbb{R}^{2}\right)}+C \varepsilon\|w\|_{L_{\gamma, \delta}^{2}\left(\mathbb{R}^{2}\right)}+C_{\varepsilon}\|w\|_{L^{2}\left(B_{\bar{R}_{\varepsilon}}\right)},
$$


where $C>0$ does not depend on $\varepsilon$. The result then follows at once, choosing $\varepsilon$ small enough so that $C \varepsilon \leq 1 / 2$.

Thanks to the previous corollary, we are now in a position to prove the following key result, which is also the main result of this section. It again enlightens the role of $S$, the set of indicial roots of $\mathcal{L}$ :

Proposition 5.2. Assume that $\gamma \in \mathbb{R} \backslash S$ and

$$
\delta^{2}+\gamma^{2}<\alpha^{2}
$$

Then, the operator

$$
\begin{aligned}
& \mathfrak{A}_{\gamma, \delta}: \quad L_{\gamma, \delta}^{2}\left(\mathbb{R}^{2}\right) \quad \longrightarrow \quad L_{\gamma, \delta}^{2}\left(\mathbb{R}^{2}\right), \\
& w \quad \longmapsto \quad \mathfrak{L} w
\end{aligned}
$$

is Fredholm. In addition $\mathfrak{A}_{\gamma, \delta}$ is injective if and only if $\mathfrak{A}_{-\gamma,-\delta}$ is surjective.

Proof. These are classical results of Fredlhom theory, and their proofs follow those of analogous statements in the compact setting, together with intensive use of Corollary 5.1. For example, to prove that the kernel of $\mathfrak{A}_{\gamma, \delta}$ is finite dimensional, we consider

$$
\mathcal{B}_{1}:=\left\{w \in \operatorname{Ker} \mathfrak{A}_{\gamma, \delta}:\|w\|_{L^{2}\left(B_{\bar{R}}\right)} \leq 1\right\},
$$

the unit ball of $\operatorname{Ker} \mathfrak{A}_{\gamma, \delta}$ using the norm of $L^{2}\left(B_{\bar{R}}\right)$. Making use of the result of Corollary 5.1 together with elliptic estimates, we see that this set is also bounded in $W_{\gamma, \delta}^{2,2}\left(\mathbb{R}^{2}\right)$ and Sobolev embedding implies that it is compact in $L^{2}\left(B_{\bar{R}}\right)$. In particular, this implies that $\operatorname{Ker} \mathfrak{A}_{\gamma, \delta}$ is finite dimensional.

The proof that $\mathfrak{A}_{\gamma, \delta}$ is closed is again based on Corollary [5.1 using similar arguments. For details, we refer the reader to [22]. Finally, the last statement follows from standard results on unbounded operators (see for example [4]) together with the natural identification of the dual of $L_{\gamma, \delta}^{2}\left(\mathbb{R}^{2}\right)$ with $L_{-\gamma,-\delta}^{2}\left(\mathbb{R}^{2}\right)$.

\section{Linear Decomposition Lemma for $\mathfrak{L}$}

In this section, we pursue our investigations of the properties of the operator $\mathfrak{A}_{\gamma, \delta}$. We assume that $\mathfrak{L}$ is the linearized operator about an element $u \in \mathcal{M}_{2 k}$ such that $u-u_{\lambda} \in W_{-\bar{\gamma},-\bar{\delta}}^{2,2}\left(\mathbb{R}^{2}\right)$, for some $\bar{\gamma}, \bar{\delta}>0$ satisfying (2.14).

First, we derive a Linear Decomposition Lemma for the operator $\mathfrak{L}$ in the spirit of the one we have obtained in $\$ 4.3$ for the operator $\mathcal{L}$ and we use this result to compute the dimension of the kernel of the operator $\mathfrak{A}_{\gamma, \delta}$.

For $j=1, \ldots, 2 k$, it is convenient to define the vector fields

$$
X_{j}(\mathrm{x}):=\mathbb{I}_{j}(\mathrm{x}) \mathrm{e}_{j}^{\perp}
$$

and

$$
Y_{j}(\mathrm{x}):=\mathbb{I}_{j}(\mathrm{x})\left(\mathrm{e}_{j} \cdot\left(\mathrm{x}-\mathrm{x}_{j}\right) \mathrm{e}_{j}^{\perp}-\mathrm{e}_{j}^{\perp} \cdot\left(\mathrm{x}-\mathrm{x}_{j}\right) \mathrm{e}_{j}\right) .
$$

To have a better understanding of these two vector fields, observe that the vector fields

$$
\stackrel{\circ}{X}_{j}(\mathrm{x}):=\mathrm{e}_{j}^{\perp}
$$

and

$$
\stackrel{\circ}{Y}_{j}(\mathrm{x}):=\mathrm{e}_{j} \cdot\left(\mathrm{x}-\mathrm{x}_{j}\right) \mathrm{e}_{j}^{\perp}-\mathrm{e}_{j}^{\perp} \cdot\left(\mathrm{x}-\mathrm{x}_{j}\right) \mathrm{e}_{j}
$$


are the Killing vector fields associated, respectively, to translations in the direction of the vector $\mathrm{e}_{j}^{\perp}$ and rotation about $\mathrm{x}_{j}$, expressed in terms of the orthonormal basis $\left\{\mathrm{e}_{j}^{\perp}, \mathrm{e}_{j}\right\}$.

Finally, we define the deficiency space

$$
\mathfrak{D}:=\operatorname{Span}\left\{\mathrm{d} u\left(X_{j}\right), \mathrm{d} u\left(Y_{j}\right): j=1, \ldots, 2 k\right\} .
$$

Since we assume that $u-u_{\lambda} \in W_{-\bar{\gamma},-\bar{\delta}}^{2,2}\left(\mathbb{R}^{2}\right)$, we have

$$
\begin{aligned}
& \mathrm{d} u\left(X_{j}\right)(\mathbf{x})-\partial_{x} u_{0}\left(\left(\mathbf{x}-\mathbf{x}_{j}\right) \cdot \mathbf{e}_{j}^{\perp}\right) \in W_{-\gamma,-\delta}^{1,2}\left(\mathbb{R}^{2}\right), \\
& \mathrm{d} u\left(Y_{j}\right)-\mathbf{e}_{j} \cdot\left(\mathbf{x}-\mathbf{x}_{j}\right) \partial_{x} u_{0}\left(\left(\mathbf{x}-\mathbf{x}_{j}\right) \cdot \mathbf{e}_{j}^{\perp}\right) \in W_{-\gamma,-\delta}^{1,2}\left(\mathbb{R}^{2}\right),
\end{aligned}
$$

for any $\gamma \in(0, \bar{\gamma})$ and $\delta \in(0, \bar{\delta})$.

The following result is a consequence of the result of Lemma 4.2 .

Proposition 6.1. Assume that $\gamma \in\left(0, \sqrt{\mu_{1}}\right)$ and $\delta>0$ are fixed so that

$$
\gamma^{2}+\delta^{2}<\alpha^{2}
$$

and $\delta \in(0, \bar{\delta}), \gamma \in(0, \bar{\gamma})$. We further assume that $v \in L_{-\gamma,-\delta}^{2}\left(\mathbb{R}^{2}\right)$ and $w \in$ $L_{\gamma, \delta}^{2}\left(\mathbb{R}^{2}\right)$ are solutions of

$$
\mathfrak{L} w=v .
$$

Then

$$
w \in L_{-\gamma,-\delta}^{2}\left(\mathbb{R}^{2}\right) \oplus \mathfrak{D}
$$

and

$$
\|w\|_{L_{-\gamma,-\delta}^{2}\left(\mathbb{R}^{2}\right) \oplus \mathfrak{D}} \leq C\left(\|v\|_{L_{-\gamma,-\delta}^{2}\left(\mathbb{R}^{2}\right)}+\|w\|_{L_{\gamma, \delta}^{2}\left(\mathbb{R}^{2}\right)}\right),
$$

for some constant $C>0$ only depending on $\delta$ and $\gamma$.

Proof. The proof is decomposed into two steps.

Step 1 . We begin by showing that in the angular sectors which do not contain the ends $\lambda_{j}^{+}$, the function $w$ grows exponentially at a slightly slower rate than suggested by the fact that it belongs to $L_{\gamma, \delta}^{2}\left(\mathbb{R}^{2}\right)$. This uses the fact that, away from the $\lambda_{j}^{+}$, the linearized operator $\mathfrak{L}$ is strongly coercive. To state this precisely we use the notation introduced in the proof of Proposition 5.1

Since we work with one sector at a time, we may well assume that

$$
\lambda_{j+\frac{1}{2}}^{+} \cap\left(\mathbb{R}^{2} \backslash B_{R}\right)=\{\mathrm{x}=(0, y): y \geq R\}
$$

(this can always be achieved after a suitable rigid motion) and that the image of the angular sector $\Omega_{j+\frac{1}{2}} \cap\left(\mathbb{R}^{2} \backslash B_{R}\right)$ under this rigid motion is the angular sector

$$
K_{R, \beta}:=\{\mathrm{x}=(x, y): \beta|x| \leq y\} \cap\left(\mathbb{R}^{2} \backslash B_{R}\right),
$$

for some $\beta>0$. Likewise the image of $\Omega_{j+\frac{1}{2}}^{\prime} \cap\left(\mathbb{R}^{2} \backslash B_{R}\right)$ is the sector $K_{R, \beta / 2}$. Note that if $\theta_{j+1}-\theta_{j}$ is the oriented angle between $\lambda_{j}^{+}$and $\lambda_{j+1}^{+}$, then

$$
\cot \left(\frac{\theta_{j+1}-\theta_{j}}{2}\right)=\frac{1}{\beta} \text {. }
$$

We define

$$
\mathrm{e}_{ \pm \beta}:=\frac{(1, \pm \beta)}{\sqrt{1+\beta^{2}}}, \quad \mathrm{e}_{ \pm \beta}^{\perp}:=\frac{(\mp \beta, 1)}{\sqrt{1+\beta^{2}}}
$$


and we let $\mathrm{x}_{R}^{ \pm}$be the points of intersection of the nodal lines $y= \pm \beta x$ with $B_{R}$. Consider the functions $G_{\varepsilon}^{ \pm}$defined by

$$
G_{\varepsilon}^{ \pm}(\mathrm{x}):=\left(\cosh \left(\mathrm{e}_{ \pm \beta} \cdot\left(\mathrm{x}-\mathrm{x}_{R}^{ \pm}\right)\right)\right)^{\gamma}\left(\cosh \left(\mathrm{e}_{ \pm \beta}^{\perp} \cdot\left(\mathrm{x}-\mathrm{x}_{R}^{ \pm}\right)\right)\right)^{\delta-\varepsilon} .
$$

Choosing $M>0$ large enough and $\varepsilon>0$ sufficiently small (so that $\alpha^{2}-\gamma^{2}-(\delta-$ $\varepsilon)^{2}>0$ ), we find that the function

$$
w_{M, \varepsilon}=M\left(G_{\varepsilon}^{+}+G_{\varepsilon}^{-}\right)
$$

is a positive supersolution for the equation $\mathfrak{L} w=v$ in the sector $K_{R, \beta}$. Then, by the maximum principle and elementary geometric manipulations, we see that there exist $M>0$ and $\varepsilon^{\prime} \in(0, \varepsilon)$ such that

$$
\begin{aligned}
|w(\mathrm{x})| \leq & M\left(\cosh \left(\mathrm{e}_{+\beta} \cdot\left(\mathrm{x}-\mathrm{x}_{R}^{+}\right)\right)\right)^{\gamma-\varepsilon^{\prime}}\left(\cosh \left(\mathrm{e}_{+\beta}^{\perp} \cdot\left(\mathrm{x}-\mathrm{x}_{R}^{+}\right)\right)\right)^{\delta-\varepsilon^{\prime}} \\
& +M\left(\cosh \left(\mathrm{e}_{-\beta} \cdot\left(\mathrm{x}-\mathrm{x}_{R}^{-}\right)\right)\right)^{\gamma-\varepsilon^{\prime}}\left(\cosh \left(\mathrm{e}_{-\beta}^{\perp} \cdot\left(\mathrm{x}-\mathrm{x}_{R}^{-}\right)\right)\right)^{\delta-\varepsilon^{\prime}}
\end{aligned}
$$

in the sector $K_{R, \beta / 2}$. Thus, at least as far as the sector $K_{R, \beta / 2}$ is concerned, the function $w$ is in a better space than the one predicted initially by the fact that we know that $w \in L_{\gamma, \delta}^{2}\left(\mathbb{R}^{2}\right)$.

Step 2. Again, applying a suitable rigid motion, we may assume that the nodal line $\lambda_{j}^{+}$is contained in the $y$-axis, $y>0$. In this case, the lines bisecting the angles between $\lambda_{j}^{+}$and $\lambda_{j+1}^{+}$and $\lambda_{j}^{+}$and $\lambda_{j-1}^{+}$become respectively

$$
L_{1}:=\left\{(x, y): y=-\beta_{1} x, x<0\right\} \quad \text { and } \quad L_{2}:=\left\{(x, y): y=\beta_{2} x, \quad x>0\right\} \text {, }
$$

where $\beta_{1}, \beta_{2}>0$. We denote by $\tilde{A}_{R}$ the sector which is outside $B_{R}$ and bounded by these lines. We consider a smooth cutoff function $\tilde{\mathbb{I}}$ whose support is equal to $\tilde{A}_{R}$ and such that:

(i) $\tilde{\mathbb{I}}(x, y)=1$ whenever $y<\max \left(-\beta_{1} x, \beta_{2} x\right)$,

(ii) $\tilde{\mathbb{I}}(x, y)=0$, whenever $y>\max \left(-2 \beta_{1} x, 2 \beta_{2} x\right)$.

The function $\tilde{w}:=\tilde{\mathbb{I}} w$ solves the equation

$$
\left(-\Delta+F^{\prime \prime}\left(u_{0}\right)\right) \tilde{w}=\tilde{\mathbb{I}} v-[\Delta, \tilde{\mathbb{I}}] w+\left(F^{\prime \prime}\left(u_{0}\right)-F^{\prime \prime}(u)\right) \tilde{\mathbb{I}} w .
$$

This equation can now be considered as an equation in the whole $\mathbb{R}^{2}$ whose righthand side belongs to $\mathbb{L}_{\gamma-\varepsilon^{\prime \prime}, \delta-\varepsilon^{\prime \prime}}^{2}\left(\mathbb{R}^{2}\right)$, for some $\varepsilon^{\prime \prime}>0$. This latter fact follows from (6.6) together with elliptic estimates. Therefore, Lemma 4.2 and the discussion at the end of $\S 4$ can be used to prove that

$$
\tilde{w} \in L_{\gamma-\varepsilon^{\prime \prime}, \delta-\varepsilon^{\prime \prime}}^{2}\left(\mathbb{R}^{2}\right) \oplus \mathbb{D} .
$$

From this and considering all other ends in a similar manner, we get the existence of $\bar{\varepsilon}>0$ such that

$$
w \in L_{\gamma-\bar{\varepsilon}, \delta-\bar{\varepsilon}}^{2}\left(\mathbb{R}^{2}\right) \oplus \mathfrak{D} .
$$

This argument can now be iterated to conclude, after finitely many steps, that (6.5) holds and the proof is complete.

We introduce the following:

Definition 6.1. A solution $u \in \mathcal{M}_{2 k}$ is said to be nondegenerate if the linear operator $\mathfrak{A}_{-\gamma, \delta}$, associated to $-\Delta+F^{\prime \prime}(u)$, is injective for some $\gamma \in\left(0, \sqrt{\mu}_{1}\right)$ and some $\gamma \in \mathbb{R}$ satisfying $\gamma^{2}+\delta^{2}<\alpha^{2}$. 
Before we proceed, there is a very important remark which is due. Following the proof of Proposition 6.1. one checks that, if the operator $\mathfrak{A}_{-\gamma, \delta}$ is injective for some $\delta \in\left(0, \sqrt{\mu}_{1}\right)$ and some $\delta \in \mathbb{R}$ satisfying $\gamma^{2}+\delta^{2}<\alpha^{2}$, then it is injective for any $\delta \in\left(0, \sqrt{\mu}_{1}\right)$ and any $\delta \in \mathbb{R}$ such that $\gamma^{2}+\delta^{2}<\alpha^{2}$.

For all $\gamma, \delta>0$, we define the operator

$$
\begin{aligned}
\tilde{\mathfrak{A}}_{-\gamma,-\delta}: \quad L_{-\gamma,-\delta}^{2}\left(\mathbb{R}^{2}\right) \oplus \mathfrak{D} & \longrightarrow L_{-\gamma,-\delta}^{2}\left(\mathbb{R}^{2}\right), \\
u & \longmapsto \mathfrak{L} u .
\end{aligned}
$$

Now, as we have already done at the end of $\S 4$, we compute the dimension of the kernel of the operator $\tilde{\mathfrak{A}}_{-\gamma,-\delta}$, using Proposition 6.1 in the same way we have used Lemma 4.2 to compute the dimension of the kernel of the operator $\tilde{\mathbb{A}}_{-\gamma, \delta}$.

Proposition 6.2. Assume that $u \in \mathcal{M}_{2 k}$ is nondegenerate and further assume that $\gamma$ and $\delta$ are fixed as in Proposition 6.1. Then the operator $\tilde{\mathfrak{A}}_{-\gamma,-\delta}$ is surjective and has a $2 k$-dimensional kernel.

Proof. As already mentioned, the proof is very close to the one already outlined at the end of $\S 4$, since $u$ is nondegenerate and, in view of the above remark, the operator $\mathfrak{A}_{-\gamma,-\delta}$ is injective. Thanks to Proposition 5.2, its adjoint $\mathfrak{A}_{\gamma, \delta}$ is surjective. In particular, for all $v \in L_{-\gamma,-\delta}^{2}\left(\mathbb{R}^{2}\right) \subset L_{\gamma, \delta}^{2}\left(\mathbb{R}^{2}\right)$, we get the existence of a $w \in L_{\gamma, \delta}^{2}\left(\mathbb{R}^{2}\right)$ that is a solution of $\mathfrak{L} w=v$. Then, the result of Proposition 6.1 implies that $w \in L_{-\gamma,-\delta}^{2}\left(\mathbb{R}^{2}\right) \oplus \mathfrak{D}$ and this proves the surjectivity of $\tilde{\mathfrak{A}}_{-\gamma,-\delta}$.

We now compute the dimension of the kernel of $\tilde{\mathfrak{A}}_{-\gamma,-\delta}$. As a starting point, observe that Proposition 6.1 also implies that

$$
\operatorname{Ker}\left(\mathfrak{A}_{\gamma, \delta}\right)=\operatorname{Ker}\left(\tilde{\mathfrak{A}}_{-\gamma,-\delta}\right),
$$

and, by duality, we have

$$
\operatorname{dim} \operatorname{Ker}\left(\mathfrak{A}_{\gamma, \delta}\right)=\operatorname{codim} \operatorname{Im}\left(\mathfrak{A}_{-\gamma,-\delta}\right) .
$$

We claim that standard arguments in linear algebra, together with the result of Proposition 6.1. imply that

$$
\operatorname{dim} \mathfrak{D}=\operatorname{dim} \operatorname{Ker}\left(\mathfrak{A}_{\gamma, \delta}\right)+\operatorname{codim} \operatorname{Im}\left(\mathfrak{A}_{-\gamma,-\delta}\right) .
$$

Indeed, the operator $\mathfrak{L}$ acting on $L_{-\gamma,-\delta}^{2}\left(\mathbb{R}^{2}\right)$ is injective and is not surjective; the codimension of its image is, by definition, equal to the codimension of $\operatorname{Im}\left(\mathfrak{A}_{-\gamma,-\delta}\right)$. Adding to the function space $L_{-\gamma,-\delta}^{2}\left(\mathbb{R}^{2}\right)$ the deficiency space $\mathfrak{D}$ makes this operator $\mathfrak{L}$ surjective but creates a kernel whose dimension is equal to the dimension of $\operatorname{Ker}\left(\mathfrak{A}_{\gamma, \delta}\right)$. Therefore the dimension of $\mathfrak{D}$ is the sum of the codimension of $\operatorname{Im}\left(\mathfrak{A}_{-\gamma,-\delta}\right)$ and the dimension of $\operatorname{Ker}\left(\mathfrak{A}_{\gamma, \delta}\right)$. The proof of the claim is complete.

From this it follows that

$$
\operatorname{dim} \operatorname{Ker}\left(\tilde{\mathfrak{A}}_{-\gamma,-\delta}\right)=\operatorname{codim} \operatorname{Im}\left(\mathfrak{A}_{-\gamma,-\delta}\right)=\frac{1}{2} \operatorname{dim} \mathfrak{D}=2 k,
$$

as claimed, since $\operatorname{dim} \mathfrak{D}=4 k$.

The formula (6.8) is usually reffered to as the relative index formula. 


\section{Refined Asymptotics, the proof of Theorem 2.1}

We use the results of the previous sections, and in particular Proposition 5.1, to prove Theorem 2.1. Thus, we assume that $u=u_{\lambda}+v \in \mathcal{M}_{2 k}$, where $v \in W^{2,2}\left(\mathbb{R}^{2}\right)$ and where $\lambda=\left(\lambda_{1}, \ldots, \lambda_{2 k}\right)$. Let us denote

$$
N(u):=-\Delta u+F^{\prime}(u) .
$$

Obviously, we can expand

$$
N(u)=N\left(u_{\lambda}\right)+\mathfrak{L}_{\lambda} v+Q(v),
$$

where the linear operator

$$
\mathfrak{L}_{\lambda}:=-\Delta+F^{\prime \prime}\left(u_{\lambda}\right)
$$

is the one which has already been defined in $\S 5$ and where the nonlinear operator

$$
Q(v):=F^{\prime}\left(u_{\lambda}+v\right)-F^{\prime}\left(u_{\lambda}\right)-F^{\prime \prime}\left(u_{\lambda}\right) v
$$

collects the nonlinear terms.

Now, if $N(u)=0$, then we find that the function $v$ solves

$$
\mathfrak{L}_{\lambda} v=-N\left(u_{\lambda}\right)-Q(v) .
$$

As we will see later, the function $N\left(u_{\lambda}\right)$ belongs to $L_{-\gamma,-\delta}^{2}\left(\mathbb{R}^{2}\right)$ for some $\gamma, \delta>0$ close enough to 0. This, together with the Linear Decomposition Lemma and the quadratic nature of the term $Q(v)$, strongly suggests that we should be able to prove that the function $v$ belongs to $\left(W_{-\gamma,-\delta}^{2,2}\left(\mathbb{R}^{2}\right) \oplus \mathfrak{D}\right) \cap W^{2,2}\left(\mathbb{R}^{2}\right)$. The fact that the elements of the deficiency space $\mathfrak{D}$ do not belong to $W^{2,2}\left(\mathbb{R}^{2}\right)$ then implies that $v \in W_{-\gamma,-\delta}^{2,2}\left(\mathbb{R}^{2}\right)$. Unfortunately, the situation turns out to be slightly more complicated since, to begin with, the only information we have to start the process is that $v \in W^{2,2}\left(\mathbb{R}^{2}\right)$ and hence we do not have any exponential decay for the function $v$ and, as a consequence, we do not have any decay property for the term $Q(v)$. In summary, a simple-minded bootstrap argument cannot be applied to carry out the above scheme.

On the other hand, if we knew that $v$ had some exponential decay at infinity, then right away a bootstrap argument would give us the optimal exponential decay for the function $v$, comparable with the one of the term $N\left(u_{\lambda}\right)$. So the main issue is to be able to start the bootstrap process. To this end, we use a scaling argument inspired by an idea of L. Simon. In [12] a similar argument was used to derive some refined asymptotic properties for constant scalar curvature metrics with isolated singularities. One of the crucial ingredients in the proof is the balancing formula (11.1) which is used to derive (2.16) and (2.17).

Lemma 7.1. Assume that $\bar{\gamma}>0$ and $\bar{\delta}>0$ are fixed such that $\bar{\gamma}^{2}+\bar{\delta}^{2}<\alpha^{2}$ and

$$
\bar{\gamma} \cot \left(\frac{\theta_{j+1}-\theta_{j}}{2}\right)+\bar{\delta}<\alpha,
$$

for $j=1, \ldots, 2 k$ (recall that $\theta_{j}$ is the angle which defines the oriented half-line $\lambda_{j}^{+}$). Then,

$$
\left|N\left(u_{\lambda}\right)\right| \leq C \Gamma_{-\gamma,-\delta},
$$

for any $\gamma \in(0, \bar{\gamma})$ and $\delta \in(0, \bar{\delta})$, where $\Gamma_{-\gamma,-\delta}$ is the weight function defined in (2.10). 
Proof. We denote for the sake of brevity $u_{0, j}:=(-1)^{j} u_{0}\left(\operatorname{dist}^{s}\left(\cdot, \lambda_{j}\right)\right)$. To begin with, we write

$$
N\left(u_{\lambda}\right)=\sum_{j=1}^{2 k}\left[-\Delta, \mathbb{I}_{j}\right] u_{0, j}+F^{\prime}\left(u_{\lambda}\right)-\sum_{j=1}^{2 k} \mathbb{I}_{j} F^{\prime}\left(u_{0, j}\right) .
$$

To estimate the first of the terms on the right-hand side, we consider the set

$$
O_{\ell}:=\left\{\mathrm{x} \in \mathbb{R}^{2}:\left(\mathbb{I}_{\ell}+\mathbb{I}_{\ell+1}\right)(\mathrm{x})=1\right\},
$$

for some $1 \leq \ell \leq 2 k$. Then, using the fact that $\mathbb{I}_{\ell}+\mathbb{I}_{\ell+1} \equiv 1$ in $O_{\ell}$, we can write

$$
\sum_{j=1}^{2 k}\left[-\Delta,(-1)^{j} \mathbb{I}_{j}\right] u_{0, j}=-2 \nabla\left(u_{0, \ell}+u_{0, \ell+1}\right) \cdot \nabla \mathbb{I}_{\ell}-\left(u_{0, \ell}+u_{0, \ell+1}\right) \Delta \mathbb{I}_{\ell},
$$

in $O_{\ell}$. We recall now that the heteroclinic solution $u_{0}$, which is odd, satisfies

$$
0 \leq 1-u_{0}(x) \leq C(\cosh x)^{-\alpha},
$$

for some constant $C>0$, with similar estimates for the derivatives of $u_{0}$. Thus, we get

$$
\left|\left(u_{0, j}+u_{0, j+1}\right)(\mathrm{x})\right| \leq C \sum_{\ell=0,1} e^{-\bar{\gamma}\left(\mathrm{x}-\mathrm{x}_{j+\ell}\right) \cdot \mathrm{e}_{j+\ell}}\left(\cosh \left(\left(\mathrm{x}-\mathrm{x}_{j+\ell}\right) \cdot \mathrm{e}_{j+\ell}^{\perp}\right)\right)^{-\bar{\delta}}
$$

for $\mathrm{x} \in O_{\ell}$, provided $\bar{\gamma}>0$ and $\bar{\delta}>0$ satisfy (7.3). Since analogous estimates hold for the term involving the gradient of $u_{0, j}+u_{0, j+1}$, we conclude that

$$
\left|\sum_{j=1}^{2 k}\left[-\Delta, \mathbb{I}_{j}\right] u_{0, j}\right| \leq C \Gamma_{-\gamma,-\delta}
$$

for $\gamma \in(0, \bar{\gamma}), \delta \in(0, \bar{\delta})$. The estimates of the other term in (7.4) can be obtained using similar arguments. This completes the proof of the result.

For further use, we assume that the constant $\gamma$, which appears in Lemma 7.1 and which is used below, is also chosen so that $\gamma \in\left(0, \sqrt{\mu_{1}}\right)$. For all $R>0$, we define

$$
\mathcal{E}(R):=\max \left(e^{-a R},\left\|u-u_{\lambda}\right\|_{L^{\infty}\left(\mathbb{R}^{2} \backslash B_{R}\right)}\right),
$$

where the constant $a>0$ is chosen close enough to 0 . We now state the main result of this section.

Proposition 7.1. There exist $\bar{R}>0$ and $r_{*}>0$ such that for all $R>\bar{R}$, we have

$$
\mathcal{E}\left(R+r_{*}\right) \leq \frac{1}{2} \mathcal{E}(R) .
$$

Before we proceed with the proof of this proposition, let us explain how Theorem 2.1 follows easily from it. Indeed, assuming we have already proven this proposition, we define

$$
\bar{a}:=\frac{1}{2 r_{*}} \log 2>0,
$$

and, using (7.6), we get for any $R>\bar{R}$,

$$
\mathcal{E}(R) \leq \mathcal{E}(\bar{R}) e^{-\bar{a}(R-\bar{R})} .
$$


This readily implies that

$$
\left\|u-u_{\lambda}\right\|_{L^{\infty}\left(\mathbb{R}^{2} \backslash B_{R}\right)} \leq \mathcal{E}(\bar{R}) e^{-\bar{a}(R-\bar{R})},
$$

from which we get that $u-u_{\lambda} \in L_{-\gamma,-\delta}^{2}\left(\mathbb{R}^{2}\right)$ for some $\delta, \gamma>0$ close enough to 0 . As we have pointed out, a bootstrap argument now gives the required result.

The proof of Proposition 7.1 involves several steps. Using appropriate barrier functions, we show that in the angular sectors which do not contain the ends $\lambda_{1}^{+}, \ldots, \lambda_{2 k}^{+}$, the function $v:=u-u_{\lambda}$ decays exponentially. To state this precisely, we use once again the notation used in the proof of Proposition [5.1.

Lemma 7.2. There exist constants $a_{0}>0, \delta_{0}>0, C>0$ and $\bar{R}_{0}>0$ such that, for all $R>\bar{R}_{0}$, we have

$$
\left|u-u_{\lambda}\right| \leq C\left(e^{-a_{0}|\mathrm{x}|}+\left(e^{-a_{0}(|\mathrm{x}|-R)}+\Gamma_{0,-\delta_{0}}\right)\left\|u-u_{\lambda}\right\|_{L^{\infty}\left(\mathbb{R}^{2} \backslash B_{R}\right)}\right),
$$

in $\mathbb{R}^{2} \backslash B_{R}$, where $\Gamma_{0,-\delta_{0}}$ is the weight function defined in (2.10).

Proof. It is enough to work in one sector at a time, say for example $\Omega_{j+\frac{1}{2}}$. After a rigid motion (and a possible change of the origin), we may assume that

$$
\lambda_{j+\frac{1}{2}}^{+} \cap\left(\mathbb{R}^{2} \backslash B_{R}\right)=\{\mathrm{x}=(0, y): y \geq R\}
$$

and that the image of the angular sector $\Omega_{j+\frac{1}{2}} \cap\left(\mathbb{R}^{2} \backslash B_{R}\right)$ under this rigid motion is the angular sector

$$
K_{R, \beta}:=\{\mathrm{x}=(x, y): \beta|x| \leq y\} \cap\left(\mathbb{R}^{2} \backslash B_{R}\right),
$$

where $\beta>0$.

We define the function

$$
\begin{aligned}
G_{\sigma, \tau}(\mathrm{x}):= & \left(\varepsilon e^{\sigma(|\mathrm{x}|-R)}+e^{-\sigma(|\mathrm{x}|-R)}+e^{-\tau(y-\beta x)}+e^{-\tau(y+\beta x)}\right)\left\|u-u_{\lambda}\right\|_{L^{\infty}\left(\mathbb{R}^{2} \backslash B_{R}\right)} \\
& +\left(\varepsilon e^{\sigma|\mathrm{x}|}+e^{-\sigma|\mathrm{x}|}\right)
\end{aligned}
$$

where $\sigma, \tau>0$ are chosen close enough to 0 . Observe that $G_{\sigma, \tau}>0$ in $K_{R, \beta}$ and

$$
G_{\sigma, \tau}(\mathrm{x}) \geq\left\|u-u_{\lambda}\right\|_{L^{\infty}\left(\mathbb{R}^{2} \backslash B_{R}\right)}
$$

on $\partial K_{R, \beta}$. Moreover, we have

$$
\left(-\Delta+\frac{\alpha^{2}}{2}\right) G_{\sigma, \tau} \geq \frac{\alpha^{2}}{4} e^{-\sigma|\mathrm{x}|}
$$

in $K_{R, \beta}$ provided $R$ is chosen large enough and $\sigma, \tau>0$ are chosen close enough to 0 . We set $v:=u-u_{\lambda}$. Observe that the function $v$ satisfies the equation

$$
(-\Delta+\Phi) v+N\left(u_{\lambda}\right)=0
$$

where by definition the potential $\Phi$ is given by

$$
\Phi:=\frac{F^{\prime}(u)-F^{\prime}\left(u_{\lambda}\right)}{u-u_{\lambda}}
$$

whenever $u-u_{\lambda} \neq 0$ and

$$
\Phi:=F^{\prime \prime}\left(u_{\lambda}\right),
$$

whenever $u=u_{\lambda}$. Now, choosing $\bar{R}$ large enough, we can ensure that $\Phi \geq \alpha^{2} / 4$ in the subset of $\bar{K}_{R, \beta}$ defined by

$$
\bar{K}_{R, \beta}:=\left\{\mathrm{x} \in K_{R, \beta}: \operatorname{dist}\left(\mathrm{x}, \partial K_{R, \beta}\right) \geq \bar{R}\right\},
$$


for all $R \geq \bar{R}$. This follows at once from the fact that $u_{\lambda}$ converges uniformly to \pm 1 away from the $\lambda_{j}^{+}$and the fact that the function $u-u_{\lambda}$ tends uniformly to 0 at infinity.

Using the result of Lemma 7.1, we find that, for each $\varepsilon>0$, a multiple of $G_{\sigma, \tau}$ is a positive supersolution for problem (7.11) in the sector $K_{R, \beta}$ provided $R$ is chosen large enough and $\sigma, \tau>0$ close enough to 0 . Therefore, we conclude that

$$
|v| \leq C G_{\sigma, \tau}
$$

in $K_{R, \beta}$. The assertion of the lemma follows at once from letting $\varepsilon$ tend to 0 in this pointwise estimate.

Next, we estimate the function $v$ near the half-line $\lambda_{j}^{+}$. As already mentioned, there is no loss of generality in assuming that the half-line $\lambda_{j}^{+}$coincides with the $y$-axis, if this is not the case, this can be achieved using some appropriate rigid motion.

So, we fix $j$ and $R>0$ large enough. We define $\Omega_{j, R}$ to be the connected component of $\mathbb{R}^{2}$ which contains the half-line $\lambda_{j}^{+} \cap\left(\mathbb{R}^{2} \backslash B_{R}\right)$ and which is bounded by the half-lines $\lambda_{j-\frac{1}{2}}^{+} \cap\left(\mathbb{R}^{2} \backslash B_{R}\right), \lambda_{j+\frac{1}{2}}^{+} \cap\left(\mathbb{R}^{2} \backslash B_{R}\right)$ and $\partial B_{R}$. The angle between two consecutive ends is strictly less than $\pi$ and hence $\Omega_{j, R} \subset \mathbb{R} \times(0, \infty)$. Observe that $\Omega_{j, R}$ is close to the set $\Omega_{j}$ which has been defined in (2.5) but is not exactly equal to it. Moreover, the subscript $R$ in $\Omega_{j, R}$ is here to emphasize the role of $R$ in what follows.

We define $\check{\mathbb{I}}_{j, R}$ to be a cutoff function such that

(i) $\check{\mathbb{I}}_{j, R} \equiv 1$ in the subset of $\Omega_{j, R}$ such that $\operatorname{dist}\left(\mathrm{x}, \partial \Omega_{j, R}\right) \geq 1$,

(ii) $\check{\mathbb{I}}_{j, R} \equiv 0$ in $\mathbb{R}^{2} \backslash \Omega_{j, R}$,

(iii) $\left|\nabla^{\ell} \check{\mathbb{I}}_{j, R}\right| \leq C$, for $\ell=0,1$ and 2 .

Again, $\check{\mathbb{I}}_{j, R}$ is close to $\mathbb{I}_{j}$, which has been defined in $\S 2.1$ but is not equal to it.

Finally, we define the function

$$
v_{j, R}:=\check{\mathbb{I}}_{j, R}\left(u-u_{\lambda}\right) .
$$

Before proceeding further we state the following corollary of Lemma 7.2. From now on, we assume that the constant $a>0$ which appears in the definition of $\mathcal{E}$ given in (7.5) is chosen so that $a<a_{0}$, where $a_{0}$ is the constant given in Lemma 7.2

Corollary 7.1. There exist $\bar{R}>0$ and $r_{*}>0$ such that for each $R>\bar{R}$,

$$
\left|\left(u-u_{\lambda}\right)(\mathrm{x})\right| \leq \frac{1}{8} \mathcal{E}(R),
$$

for all $\mathrm{x} \in \mathbb{R}^{2}$ such that $\operatorname{dist}\left(\mathrm{x}, \lambda_{j}^{+}\right) \geq \bar{R}$ for all $j=1, \ldots, 2 k$, and $|\mathrm{x}| \geq R+r_{*}$.

Since $u$ is a solution of (2.1), one can check that the function $v_{j, R}$ is a solution of

$$
\begin{aligned}
\left(-\Delta+F^{\prime \prime}\left(u_{\lambda}\right)\right) v_{j, R}= & \check{\mathbb{I}}_{j, R} N\left(u_{\lambda}\right)-\left[\Delta, \check{\mathbb{I}}_{j, R}\right]\left(u-u_{\lambda}\right) \\
& -\check{\mathbb{I}}_{j, R}\left(F^{\prime}(u)-F^{\prime}\left(u_{\lambda}\right)-F^{\prime \prime}\left(u_{\lambda}\right)\left(u-u_{\lambda}\right)\right) .
\end{aligned}
$$


Since $F$ is even, so is $F^{\prime \prime}$, and hence, close to $\lambda_{j}^{+}, F^{\prime \prime}\left(u_{0}\right)$ is equal to $F^{\prime \prime}\left(u_{\lambda}\right)$. Therefore, we can rewrite (7.13) as

$$
\begin{aligned}
\left(-\Delta+F^{\prime \prime}\left(u_{0}\right)\right) v_{j, R}= & \check{\mathbb{I}}_{j, R} N\left(u_{\lambda}\right)-\left[\Delta, \check{\mathbb{I}}_{j, R}\right]\left(u-u_{\lambda}\right) \\
& +\left(F^{\prime \prime}\left(u_{0}\right)-F^{\prime \prime}\left(u_{\lambda}\right)\right) v_{j, R} \\
& -\check{\mathbb{I}}_{j, R}\left(F^{\prime}(u)-F^{\prime}\left(u_{\lambda}\right)-F^{\prime \prime}\left(u_{\lambda}\right)\left(u-u_{\lambda}\right)\right) .
\end{aligned}
$$

We denote for short

$$
\begin{gathered}
h_{j, R}^{1}:=\check{\mathbb{I}}_{j, R} N\left(u_{\lambda}\right), \\
h_{j, R}^{2}:=-\left[\Delta, \check{\mathbb{I}}_{j, R}\right]\left(u-u_{\lambda}\right)+\left(F^{\prime \prime}\left(u_{0}\right)-F^{\prime \prime}\left(u_{\lambda}\right)\right) v_{j, R},
\end{gathered}
$$

and

$$
h_{j, R}^{3}:=-\check{\mathbb{I}}_{j, R}\left(F^{\prime}(u)-F^{\prime}\left(u_{\lambda}\right)-F^{\prime \prime}\left(u_{\lambda}\right)\left(u-u_{\lambda}\right)\right),
$$

so that (7.14) becomes

$$
\left(-\Delta+F^{\prime \prime}\left(u_{0}\right)\right) v_{j, R}=h_{j, R}^{1}+h_{j, R}^{2}+h_{j, R}^{3} .
$$

We decompose any function $f$ defined in $\mathbb{R}^{2}$ into

$$
f=f^{\|}+f^{\perp},
$$

where $f^{\|}:=\Pi_{0}(f)$ (recall that $\Pi_{0}$ has been defined in (1.6) $)$ and $f^{\perp}$ is $L^{2}(\mathbb{R})$ orthogonal to $\partial_{x} u_{0}(x)$ for all $y \in \mathbb{R}$. Starting from (7.15), we decompose $v_{j, R}=$ $v_{j, R}^{\|}+v_{j, R}^{\perp}$, and using natural notation, we obtain the system of two equations

$$
\left(-\Delta+F^{\prime \prime}\left(u_{0}\right)\right) v_{j, R}^{\|}=h_{j, R}^{1, \|}+h_{j, R}^{2, \|}+h_{j, R}^{3, \|}
$$

and

$$
\left(-\Delta+F^{\prime \prime}\left(u_{0}\right)\right) v_{j, R}^{\perp}=h_{j, R}^{1, \perp}+h_{j, R}^{2, \perp}+h_{j, R}^{3, \perp} .
$$

We now estimate each term in the decomposition of $v_{j, R}$. First, we have:

Lemma 7.3. There exist constants $a_{1}>0, C>0$ and $\delta_{1}>0$ such that the following pointwise estimate holds:

$\left|v_{j, R}^{\perp}\right| \leq C\left(e^{-a_{1}|\mathrm{x}|}+e^{-a_{1}(|\mathrm{x}|-R)}\left\|u-u_{\lambda}\right\|_{L^{\infty}\left(\mathbb{R}^{2} \backslash B_{R}\right)}+\Gamma_{0,-\delta_{1}}\left\|u-u_{\lambda}\right\|_{L^{\infty}\left(\mathbb{R}^{2} \backslash B_{R}\right)}^{2}\right)$.

Proof. This estimate follows at once from Remark 4.2, which ensures that, since we are working in the $L^{2}$-orthogonal complement of the space of functions of the form $f(y) \partial_{x} u_{0}(x)$, we can choose $\gamma \in\left(-\sqrt{\mu_{1}}, \sqrt{\mu_{1}}\right)$ in Proposition 4.1 to estimate $v_{j, R}^{\perp}$ in terms of the norms of $h_{j, R}^{1, \perp}, h_{j, R}^{2, \perp}$ and $h_{j, R}^{3, \perp}$.

To estimate the first term $h_{j, R}^{1, \perp}$, we make use of Lemma 7.1 and, applying Proposition 4.1 with $\delta=-\hat{\delta}, \gamma=-\hat{\gamma}$ we get a contribution to the estimate of $v_{j, R}^{\perp}$ which is bounded by a constant times $e^{-a_{1}|\mathrm{x}|}$ provided $a_{1}>0$ is chosen close enough to 0 (the constants $\hat{\delta}$ and $\hat{\gamma}$ are the constants satisfying the hypotheses of Lemma 7.1 and $\left.\hat{\gamma} \in\left(0, \sqrt{\mu}_{1}\right)\right)$. To estimate the second term $h_{j, R}^{2, \perp}$, we use the result of Lemma 7.2 which ensures that $u-u_{\lambda}$ and $u_{\lambda}-u_{0}$, restricted to the support of $h_{j, R}^{2, \perp}$, decay exponentially at infinity. Then, applying Proposition 4.1 with some $\gamma<0$ close enough to 0 and $\delta=0$, we obtain that this term contributes to the estimate of $v_{j, R}^{\perp}$ by a constant times $e^{-\gamma R} \Gamma_{-\gamma, 0}\left\|u-u_{\lambda}\right\|_{L^{\infty}\left(\mathbb{R}^{2} \backslash B_{R}\right)}^{2}$. Observe that the main error comes from the action of the cutoff function when $\mathrm{x} \in \Omega_{j, R}$ satisfies $|\mathrm{x}| \in[R, R+1]$. 
Finally, to estimate the third term $h_{j, R}^{3, \perp}$, we apply Proposition 4.1 with $\gamma=0$ and $\delta<0$ close enough to 0 . The details are left to the reader.

Assuming that $a>0$, the constant which appears in the definition of $\mathcal{E}$, which is given in (7.5), is chosen so that $a<a_{1}$, where $a_{1}$ is the constant given in Lemma 7.3 . The last lemma implies that:

Corollary 7.2. There exist $r_{*}>0, \bar{R}>0$ and $\zeta>0$ such that

$$
\sup _{|\mathrm{x}|>R+r_{*}}\left|v_{j, R}^{\perp}\right| \leq \frac{1}{8} \mathcal{E}(R)
$$

provided $\mathcal{E}(R) \leq \zeta$ and $R \geq \bar{R}$.

The next step is to understand the solution of

$$
\left(-\Delta+F^{\prime \prime}\left(u_{0}\right)\right) v_{j, R}^{\|}=h_{j, R}^{1, \|}+h_{j, R}^{2, \|}+h_{j, R}^{3, \|}
$$

If we write

$$
v_{j, R}^{\|}(x, y)=\phi_{j, R}(y) \partial_{x} u_{0}(x) \quad \text { and } \quad h_{j, R}^{\ell, \|}(x, y)=\psi_{j, R}^{\ell}(y) \partial_{x} u_{0}(x),
$$

for $\ell=1,2,3$, we find that $\phi_{j, R}$ is a solution of

$$
-\partial_{y}^{2} \phi_{j, R}=\psi_{j, R}^{1}+\psi_{j, R}^{2}+\psi_{j, R}^{3}
$$

Since $v_{j, R}$ has support in $\mathbb{R} \times(R, \infty)$, we conclude that $\phi_{j, R}$ has support in $(R, \infty)$. In particular,

$\phi_{j, R}(y)=\kappa^{b}+\kappa^{\sharp}(y-R)-\int_{y}^{+\infty} \int_{t}^{+\infty}\left(\psi_{j, R}^{1}(s)+\psi_{j, R}^{2}(s)\right) d s d t-\int_{0}^{y} \int_{0}^{t} \psi_{j, R}^{3}(s) d s d t$,

for some $\kappa^{b}, \kappa^{\sharp} \in \mathbb{R}$.

We have the following:

Lemma 7.4. There exist constants $a_{2}>0$ and $C>0$ such that

$$
\left|\int_{y}^{+\infty} \int_{t}^{+\infty}\left(\psi_{j, R}^{1}(s)+\psi_{j, R}^{2}(s)\right) d s d t\right| \leq C\left(e^{-a_{2} y}+e^{-a_{2}(y-R)}\left\|u-u_{\lambda}\right\|_{L^{\infty}\left(\mathbb{R}^{2} \backslash B_{R}\right)}\right)
$$

and

$$
\left|\int_{0}^{y} \int_{0}^{t} \psi_{j, R}^{3}(s) d s d t\right| \leq C\left\|u-u_{\lambda}\right\|_{L^{\infty}\left(\mathbb{R}^{2} \backslash B_{R}\right)}^{2}\left(1+(\max \{0, y-R\})^{2}\right),
$$

for all $y \geq 0$.

Proof. The proof is a simple consequence of Lemma 7.1 and Lemma 7.2 .

We now estimate the parameters $\kappa^{b}$ and $\kappa^{\sharp}$. We start with:

Lemma 7.5. There exists a constant $C>0$ such that the following estimates hold:

$$
\left|\kappa^{\sharp}\right| \leq C\left(e^{-a_{2} R}+\left\|u-u_{\lambda}\right\|_{L^{\infty}\left(\mathbb{R}^{2} \backslash B_{R}\right)}\right)^{1 / 2}\left\|u-u_{\lambda}\right\|_{L^{\infty}\left(\mathbb{R}^{2} \backslash B_{R}\right)},
$$

provided $\left\|u-u_{\lambda}\right\|_{L^{\infty}\left(\mathbb{R}^{2} \backslash B_{R}\right)} \leq 1$. 
Proof. Using the fact that $v_{j, R}^{\|}$is bounded by a constant times $\left\|u-u_{\lambda}\right\|_{L^{\infty}\left(\mathbb{R}^{2} \backslash B_{R}\right)}$ together with the result of Lemma 7.4, we find

$$
\left|\kappa^{b}\right| \leq C\left(e^{-a_{2} R}+\left\|u-u_{\lambda}\right\|_{L^{\infty}\left(\mathbb{R}^{2} \backslash B_{R}\right)}\right),
$$

provided $\left\|u-u_{\lambda}\right\|_{L^{\infty}\left(\mathbb{R}^{2} \backslash B_{R}\right)} \leq 1$. Once this crude estimate is obtained, we can obtain some precise estimate for $\kappa^{\sharp}$. To this aim, we again use the fact that $v_{j, R}^{\|}$is bounded by a constant times $\left\|u-u_{\lambda}\right\|_{L^{\infty}\left(\mathbb{R}^{2} \backslash B_{R}\right)}$ and using the result of Lemma 7.4 , we get

$$
(y-R)\left|\kappa^{\sharp}\right| \leq C\left(e^{-a_{2} R}+\left\|u-u_{\lambda}\right\|_{L^{\infty}\left(\mathbb{R}^{2} \backslash B_{R}\right)}+(y-R)^{2}\left\|u-u_{\lambda}\right\|_{L^{\infty}\left(\mathbb{R}^{2} \backslash B_{R}\right)}^{2}\right),
$$

for all $y \geq R$ (here we also use the fact that we assume that $\left\|u-u_{\lambda}\right\|_{L^{\infty}\left(\mathbb{R}^{2} \backslash B_{R}\right)} \leq 1$ ). In other words, for all $X \geq 0$ we have

$$
C\left\|u-u_{\lambda}\right\|_{L^{\infty}\left(\mathbb{R}^{2} \backslash B_{R}\right)}^{2} X^{2}-\left|\kappa^{\sharp}\right| X-C\left(e^{-a_{2} R}+\left\|u-u_{\lambda}\right\|_{L^{\infty}\left(\mathbb{R}^{2} \backslash B_{R}\right)}\right) \geq 0 .
$$

Thus the discriminant of the quadratic polynomial must be nonpositive. In particular, this implies that

$$
\left|\kappa^{\sharp}\right| \leq C\left(e^{-a_{2} R}+\left\|u-u_{\lambda}\right\|_{L^{\infty}\left(\mathbb{R}^{2} \backslash B_{R}\right)}\right)^{1 / 2}\left\|u-u_{\lambda}\right\|_{L^{\infty}\left(\mathbb{R}^{2} \backslash B_{R}\right)},
$$

and this completes the proof of the estimate.

In order to derive an estimate for $\kappa^{b}$, we invoke the balancing formula (11.1), which follows from Lemma 11.1. Given any Killing vector field $X$ and any compact $\Omega \subset \mathbb{R}^{2}$, this balancing formula reads

$$
\int_{\partial \Omega}\left(\left(\frac{1}{2}|\nabla u|^{2}+F(u)\right) X-X(u) \nabla u\right) \cdot \nu d s=0,
$$

where $\nu$ is an outward-pointing unit normal vector field to $\partial \Omega$. Of interest is the case where $X$ is the vector field

$$
X_{\tau}:=x \partial_{y}-y \partial_{x}
$$

which is the vector field which generates the group of rotations centered at the origin.

Let us briefly digress and consider the case where the function $u$, a solution of (2.1), is defined on $\mathbb{R} \times(0, \infty)$ and can be expanded as

$$
u(x, y)=u_{0}(x)+\kappa \partial_{x} u_{0}(x)+v(x, y),
$$

where $\kappa \in \mathbb{R}$ is a small constant and where the function $v$ is also small (in a sense to be made precise). Then, (7.16) implies that

$$
\int_{y=y_{0}}\left(\left(\frac{1}{2}|\nabla u|^{2}+F(u)\right) X-X(u) \nabla u\right) \cdot \nu d s
$$

does not depend on $y_{0}$. On the one hand, when $u$ is replaced by $u_{0}$, this quantity is equal to 0 ; hence, assuming that $u$ converges fast enough to $u_{0}$ as $y$ tends to infinity and letting $y_{0}$ tend to $\infty$, we find that

$$
\int_{y=y_{0}}\left(\left(\frac{1}{2}|\nabla u|^{2}+F(u)\right) X-X(u) \nabla u\right) \cdot \nu d s=0 .
$$


On the other hand, inserting $u=u_{0}+\kappa \partial_{x} u_{0}+v$ in this equality and assuming that $\kappa$ and $v$ are small, we get

$$
\begin{aligned}
\int_{y=y_{0}}\left(\left(\frac{1}{2}|\nabla u|^{2}+F(u)\right) X-X(u) \nabla u\right) \cdot \nu d s= & \kappa \int_{\mathbb{R}}\left(\frac{1}{2}\left|\partial_{x} u_{0}\right|^{2}+F\left(u_{0}\right)\right) d x \\
& +\mathcal{O}\left(\kappa^{2}\right)+\mathcal{O}(\|v\|) .
\end{aligned}
$$

Hence, we conclude that

$$
\kappa \int_{\mathbb{R}}\left(\frac{1}{2}\left|\partial_{x} u_{0}\right|^{2}+F\left(u_{0}\right)\right) d x+\mathcal{O}\left(\kappa^{2}\right)+\mathcal{O}(\|v\|)=0
$$

and this immediately implies that $\kappa=\mathcal{O}(\|v\|)$. This is the idea that we will implement to derive a precise estimate for the constant $\kappa^{b}$.

Lemma 7.6. For all $\varepsilon>0$, there exists $a_{3}>0$ and there exist $\zeta_{\varepsilon}>0$ and $\bar{R}_{\varepsilon}>0$ such that

$$
\left|\kappa^{b}\right| \leq C e^{-a_{3} R}+\varepsilon\left\|u-u_{\lambda}\right\|_{L^{\infty}\left(\mathbb{R}^{2} \backslash B_{R}\right)},
$$

provided $R \geq \bar{R}_{\varepsilon}$ and $\left\|u-u_{\lambda}\right\|_{L^{\infty}\left(\mathbb{R}^{2} \backslash B_{R}\right)} \leq \zeta_{\varepsilon}$.

Proof. Since we have assumed that the half-line $\lambda_{j}^{+}$coincides with half of the $y$ axis, we have $u_{\lambda}= \pm u_{0}$ close to this axis and to fix the ideas, we can assume that $u_{\lambda}=u_{0}$. Now, we define

$$
u_{j, R}:=u_{\lambda}+v_{j, R}=u+\left(1-\check{\mathbb{I}}_{j, R}\right)\left(u_{\lambda}-u\right) .
$$

We set

$$
h_{j, R}:=-\Delta u_{j, R}+F^{\prime}\left(u_{j, R}\right)=-\Delta\left(u-u_{j, R}\right)+\left(F^{\prime}\left(u_{j, R}\right)-F^{\prime}(u)\right) .
$$

In this case, (7.16) has to be replaced by

$$
\int_{\partial \Omega}\left(\left(\frac{1}{2}\left|\nabla u_{j, R}\right|^{2}+F\left(u_{j, R}\right)\right) X-X\left(u_{j, R}\right) \nabla u_{j, R}\right) \cdot \nu d s=\int_{\Omega} h_{j, R} X\left(u_{j, R}\right) d \mathrm{x} .
$$

Writing $u_{j, R}=u_{0}+w$, using the fact that $u_{0}$ satisfies (7.16) and subtracting the two equations, we obtain

$$
\begin{aligned}
\int_{\partial \Omega}\left(\frac{1}{2}\left(\left|\nabla\left(u_{0}+w\right)\right|^{2}-\left|\nabla u_{0}\right|^{2}+F\left(u_{0}+w\right)-F\left(u_{0}\right)\right) X\right. & \\
& \left.-\left(X\left(u_{0}+w\right) \nabla\left(u_{0}+w\right)-X\left(u_{0}\right) \nabla u_{0}\right)\right) \cdot \nu d s=\int_{\Omega} h_{j, R} X\left(u_{j, R}\right) d \mathbf{x} .
\end{aligned}
$$

We specialize this analysis to the case where the vector field $X$ is given by $X=$ $x \partial_{y}-y \partial_{x}$ and where $\Omega:=\mathbb{R} \times\left[y_{0}, y_{1}\right]$. Letting $y_{1}$ tend to $\infty$, we get

$$
\int_{y=y_{0}}\left(\partial_{x} u_{0}\left(\partial_{x} w-\partial_{y} w\right)+\left(F\left(u_{0}+w\right)-F\left(u_{0}\right)\right)\right) d x=\int_{y \geq y_{0}} h_{j, R} X\left(u_{j, R}\right) d \mathbf{x} .
$$

We decompose

$$
u_{j, R}=u_{0}+\kappa^{b} \partial_{x} u_{0}+\tilde{w}
$$

and choose $y_{0} \geq R+1$. Thanks to the estimates already derived, we conclude, with little work, that there exists $a_{3}>0$ such that

$$
\begin{aligned}
\left|\kappa^{b}\right| \leq & C\left(e^{-a_{3} R}+e^{-a_{3}\left(y_{0}-R\right)}\left\|u-u_{\lambda}\right\|_{L^{\infty}\left(\mathbb{R}^{2} \backslash B_{R}\right)}+\left(y_{0}-R\right)^{2}\left\|u-u_{\lambda}\right\|_{L^{\infty}\left(\mathbb{R}^{2} \backslash B_{R}\right)}^{2}\right. \\
& \left.+\left(y_{0}-R\right)\left\|u-u_{\lambda}\right\|_{L^{\infty}\left(\mathbb{R}^{2} \backslash B_{R}\right)}\left(e^{-a_{2} R}+\left\|u-u_{\lambda}\right\|_{L^{\infty}\left(\mathbb{R}^{2} \backslash B_{R}\right)}\right)^{1 / 2}\right),
\end{aligned}
$$


provided $\left(y_{0}-R\right)\left\|u-u_{\lambda}\right\|_{L^{\infty}\left(\mathbb{R}^{2} \backslash B_{R}\right)} \leq 1$. At this stage, in order to derive the estimate, it is enough to choose $r_{0}:=y_{0}-R$ large enough so that

$$
C e^{-a_{3}\left(y_{0}-R\right)} \leq \frac{\varepsilon}{2}
$$

and, once this is done, we choose $\bar{R}_{\varepsilon}>0$ large enough and $\zeta_{\varepsilon}>0$ small enough so that

$$
C\left(r_{0}^{2} \zeta_{\varepsilon}+r_{0}\left(e^{-a_{2} \bar{R}_{\varepsilon}}+\zeta_{\varepsilon}\right)^{1 / 2}\right) \leq \frac{\varepsilon}{2}
$$

The proof of the result is then complete.

Assuming that the constant $a>0$ which appears in the definition of $\mathcal{E}$ given in (7.5) is chosen so that $a<\min \left(a_{2}, a_{3}\right)$, where $a_{2}, a_{3}$ are the constants given in Lemma 7.4 and Lemma 7.6, we get:

Corollary 7.3. There exist $r_{*}>0, \bar{R}>0$ and $\zeta>0$ such that

$$
\sup _{|\mathbf{x}|>R+r_{*}}\left|v_{j, R}^{\|}\right| \leq \frac{1}{8} \mathcal{E}(R)
$$

provided $\mathcal{E}(R) \leq \zeta$ and $R \geq \bar{R}$.

The result of Proposition 7.1 follows from Corollary 7.1. Corollary 7.2 and Corollary 7.3 . Observe that one can always choose $\bar{R}>0$ large enough so that $\mathcal{E}(\bar{R}) \leq \zeta$, where $\zeta$ is the least of the two constants $\zeta$ which appear in the statements of Corollary 7.2 and Corollary 7.3 .

\section{The implicit FUNCTION THEOREM}

We are now in a position to prove Theorem 2.2. To this end, we apply the implicit function theorem using the linear analysis derived in the previous sections. By now, this argument is rather standard and hence, we only outline the main points of the proof, leaving the details to the reader.

We start by defining a smooth family of diffeomorphisms of $\mathbb{R}^{2}$ as follows: Given $\mathrm{a}=\left(a_{1}, \ldots, a_{2 k}\right) \in \mathbb{R}^{2 k}$ and $\mathrm{b}=\left(b_{1}, \ldots, b_{2 k}\right) \in \mathbb{R}^{2 k}$, we define $\Phi_{\mathrm{a}, \mathrm{b}}$ to be the value, at time 1 , of the flow associated to the vector field

$$
\Xi_{\mathrm{a}, \mathrm{b}}:=\sum_{j=1}^{2 k}\left(a_{j} X_{j}+b_{j} Y_{j}\right) .
$$

Here $X_{j}, Y_{j}$ are the vector fields defined in (6.1) and (6.2). Recall that $X_{j}$ generates translations in a direction which is transversal to the $j$-th end while $Y_{j}$ generates a rotation of the $j$-th end. The map $\Phi_{\mathrm{a}, \mathrm{b}}$ is clearly a diffeomorphism provided all coefficients $a_{j}$ and $b_{j}$ are small enough. Also, observe that, in most of the sector where $\mathbb{I}_{j} \equiv 1$, the map $\Phi_{\mathrm{a}, \mathrm{b}}$ is equal to a rigid motion and hence it commutes with the Laplacian $\Delta$ in the sense that

$$
\Delta\left(w \circ \Phi_{\mathrm{a}, \mathrm{b}}\right)=(\Delta w) \circ \Phi_{\mathrm{a}, \mathrm{b}} .
$$

We choose $\gamma, \delta>0$ close enough to 0 . Given a function $v \in W_{-\gamma,-\delta}^{2,2}\left(\mathbb{R}^{2}\right)$, given $\mathrm{a} \in \mathbb{R}^{2 k}$ and $\mathrm{b} \in \mathbb{R}^{2 k}$, we define

$$
N(v, \mathrm{a}, \mathrm{b}):=\left(-\Delta\left((u+v) \circ \Phi_{\mathrm{a}, \mathrm{b}}\right)+F^{\prime}\left((u+v) \circ \Phi_{\mathrm{a}, \mathrm{b}}\right)\right) \circ \Phi_{-\mathrm{a},-\mathrm{b}},
$$


where $u \in \mathcal{M}_{2 k}$ is fixed. It is easy to check that the nonlinear map $N$ is well defined and smooth from a neighborhood of 0 in $W_{-\gamma,-\delta}^{2,2}\left(\mathbb{R}^{2}\right) \times \mathbb{R}^{2 k} \times \mathbb{R}^{2 k}$ into $L_{\gamma, \delta}^{2}\left(\mathbb{R}^{2}\right)$. This follows from the fact that $\Phi_{\mathrm{a}, \mathrm{b}}$ is a rigid motion in the regions $\mathbb{I}_{j} \equiv 1$, and in the regions where it is not, the functions involved are in $L_{-\gamma,-\delta}^{2}\left(\mathbb{R}^{2}\right)$.

Since $\Phi_{0,0}=\mathrm{Id}$, we check directly that

$$
D_{v} N_{(0,0,0)}=\mathfrak{L} .
$$

Moreover, we have

$$
\partial_{a_{j}} N_{(0,0,0)}=\mathfrak{L}\left(\mathrm{d} u\left(X_{j}\right)\right) \quad \text { and } \quad \partial_{b_{j}} N_{(0,0,0)}=\mathfrak{L}\left(\mathrm{d} u\left(Y_{j}\right)\right) .
$$

If we assume that $u \in \mathcal{M}_{2 k}$ is nondegenerate, the result of Proposition 6.2 implies that the full differential $D N_{(0,0,0)}$ (which can be naturally identified with the operator $\tilde{\mathfrak{A}}_{-\gamma,-\delta}$ ) is surjective and has a kernel whose dimension is $2 k$. The implicit function theorem then implies that, close to $v=0, \mathrm{a}=\mathrm{b}=0$, and the set of zeros of $N$ is a smooth $2 k$-dimensional manifold. This completes the proof of Theorem 2.2 .

\section{Nondegeneracy of SOlutions With NODAL SET WITH NEARLY PARALLEL COMPONENTS}

In this section, we check that, given $k \geq 1$, the set $\mathcal{M}_{2 k}$ contains at least one nondegenerate element, thus proving Proposition 2.1. We recall that for simplicity we assume here that

$$
F(u)=\frac{1}{4}\left(1-u^{2}\right)^{2}
$$

however, all the results needed to prove the proposition generalize directly to the case of a general, smooth even nonlinearity as described in (1.2). Consequently, we expect that the assertion of Proposition 2.1 holds in this more general framework as well.

The result in $\left[8\right.$ implies the existence of a family of solutions $\left\{u_{\varepsilon}\right\}_{\varepsilon \in\left(0, \varepsilon_{0}\right)} \subset \mathcal{M}_{2 k}$ whose ends are nearly parallel and are graphs over the $y$-axis for some function whose derivative is bounded by a constant times $\varepsilon$. What is more, the construction shows that $\left\{\mathrm{x} \in \mathbb{R}^{2}: \mathrm{u}_{\varepsilon}(\mathrm{x})=0\right\}$, the nodal set of $u_{\varepsilon}$, decomposes into exactly $k$ disjoint curves whose mutual distances tend to $\infty$ as $\varepsilon$ tends to 0 .

To state our result in a precise way, we assume that we are given $\mathbf{q}:=\left(q_{1}, \ldots, q_{k}\right)$, a solution of the Toda system

$$
\bar{c}_{0} q_{j}^{\prime \prime}=e^{\sqrt{2}\left(q_{j-1}-q_{j}\right)}-e^{\sqrt{2}\left(q_{j}-q_{j+1}\right)},
$$

for $j=1, \ldots, k$, where $\bar{c}_{0}:=\frac{\sqrt{2}}{24}$ and where we agree that

$$
q_{0} \equiv-\infty \quad \text { and } \quad q_{k+1} \equiv+\infty .
$$

The Toda system (9.1) is a classical example of an integrable system which has been extensively studied. It models the dynamics of finitely many mass points on the line under the influence of an exponential potential. We recall some of the results which are concerned with the solvability of (9.1) and which are needed for our purposes. We refer to [13 and [19] for the complete description of the theory (see also 21, 24, 11). Of importance for us is the fact that solutions of (9.1) can be described (almost explicitly) in terms of $2 k$ parameters. Moreover, if $\mathbf{q}$ is a solution of (9.1), then the long-term behavior (i.e. long-term scattering) of the 
$q_{j}$ at $\pm \infty$ is well understood and it is known that, for all $j=1, \ldots, k$, there exist $a_{j}^{+}, b_{j}^{+} \in \mathbb{R}, a_{j}^{-}, b_{j}^{-} \in \mathbb{R}$ and $\tau_{0}>0$, all depending on the solution $\mathbf{q}$, such that

$$
q_{j}(t)=a_{j}^{ \pm}|t|+b_{j}^{ \pm}+\mathcal{O}_{\mathcal{C} \infty(\mathbb{R})}\left(e^{-\tau_{0}|t|}\right),
$$

as $t$ tends to $\pm \infty$. Finally, $a_{j+1}^{ \pm}>a_{j}^{ \pm}$for all $j=1, \ldots, k-1$.

Given $\varepsilon>0$, we define the vector-valued function $\mathbf{q}_{\varepsilon}$, whose components are given by

$$
q_{j, \varepsilon}(x):=q_{j}(\varepsilon x)-\sqrt{2}\left(j-\frac{k+1}{2}\right) \log \varepsilon .
$$

It is easy to check that the $q_{j, \varepsilon}$ are again solutions of (9.1).

Observe that, according to the description of the asymptotic properties of the functions $q_{j}$, the graphs of the functions $q_{j, \varepsilon}$ are asymptotic to a set of $2 k$ oriented half-lines. In addition, for $\varepsilon>0$ small enough, these graphs are disjoint and in fact their mutual distance is given by $-\sqrt{2} \log \varepsilon+\mathcal{O}(1)$ as $\varepsilon$ tends to 0 .

It is convenient to agree that $\chi^{+}$(resp. $\chi^{-}$) is a smooth cutoff function defined on $\mathbb{R}$ which is identically equal to 1 for $x>1$ (resp. for $x<-1$ ) and identically equal to 0 for $x<-1$ (resp. for $x>1$ ) and additionally $\chi^{-}+\chi^{+} \equiv 1$. With these cutoff functions at hand, we define the 4-dimensional space

$$
\hat{D}:=\operatorname{Span}\left\{x \longmapsto \chi^{ \pm}(x), \quad x \longmapsto x \chi^{ \pm}(x)\right\},
$$

and, for all $\mu \in(0,1)$ and all $\tau \in \mathbb{R}$, we define the space $\mathcal{C}_{\tau}^{2, \mu}(\mathbb{R})$ of $\mathcal{C}^{2, \mu}$ functions $h$ which satisfy

$$
\|h\|_{\mathcal{C}_{\tau}^{2, \mu}(\mathbb{R})}:=\left\|(\cosh x)^{\tau} h\right\|_{\mathcal{C}^{2, \mu}(\mathbb{R})}<\infty .
$$

Keeping the above notation in mind, the following is proven in [8]:

Theorem 9.1. For all $\varepsilon>0$ sufficiently small, there exists an entire solution $u_{\varepsilon} \in \mathcal{M}_{2 k}$ of the Allen-Cahn equation

$$
\Delta u+u-u^{3}=0
$$

in $\mathbb{R}^{2}$, whose nodal set is the union of $k$ disjoint curves $\mathcal{C}_{1, \varepsilon}, \ldots, \mathcal{C}_{k, \varepsilon}$. These curves are the graphs of the functions

$$
x \longmapsto q_{j, \varepsilon}(x)+h_{j, \varepsilon}(\varepsilon x),
$$

where the functions $h_{j, \varepsilon}$ belong to $\mathcal{C}_{\tau}^{2, \mu}(\mathbb{R}) \oplus \hat{D}$ and satisfy

$$
\left\|h_{j, \varepsilon}\right\|_{\mathcal{C}_{\tau}^{2, \mu}(\mathbb{R}) \oplus \hat{D}} \leq C \varepsilon^{a} .
$$

Here the constants $C, a, \tau, \mu>0$ are all independent of $\varepsilon>0$.

In other words, given a solution of the Toda system, we can find a one-parameter family of $2 k$-ended solutions of (9.5) which depend on a small parameter $\varepsilon>0$. As $\varepsilon$ tends to 0 , the nodal sets of the solutions become close to the graphs of the functions $q_{j, \varepsilon}$.

Furthermore, using Proposition 1.1 and Proposition 5.4 in [8], one can be more precise about the description of the solution $u_{\varepsilon}$. If $\lambda_{\varepsilon}:=\left(\lambda_{1, \varepsilon}^{+}, \ldots, \lambda_{2 k, \varepsilon}^{+}\right)$denotes the affine half-lines associated to the ends of $u_{\varepsilon}$ and if $u_{\lambda_{\varepsilon}}$ denotes the associated function defined in (2.6), we know that $u_{\varepsilon}=u_{\lambda_{\varepsilon}}+v_{\varepsilon}$, where $v_{\varepsilon} \in W_{-\gamma \varepsilon,-\delta}^{2,2}\left(\mathbb{R}^{2}\right)$ for some $\delta, \gamma>0$ such that $\delta^{2}+\gamma^{2} \varepsilon^{2}<\alpha^{2}$. In addition, we have

$$
\left\|v_{\varepsilon}\right\|_{W_{-\gamma \varepsilon,-\delta}^{2,2}\left(\mathbb{R}^{2}\right)} \leq C \varepsilon^{\bar{a}} \text {, }
$$

for some $\bar{a}>0$. 
Proof of Proposition 2.1. Our goal is to show that there exists $\varepsilon_{0}>0$ such that for all $\varepsilon \in\left(0, \varepsilon_{0}\right)$ the solution $u_{\varepsilon}$ of (9.5) which is given by Theorem 9.1 is nondegenerate. We fix $\bar{\gamma}, \delta>0$ such that $\delta^{2}<\alpha^{2}$. We claim that, for $\varepsilon>0$ sufficiently small, the unique solution of the equation

$$
-\left(\Delta-F^{\prime \prime}\left(u_{\varepsilon}\right)\right) w=0,
$$

which belongs to $L_{-\bar{\gamma} \varepsilon,-\delta}^{2}\left(\mathbb{R}^{2}\right)$, is $w \equiv 0$ (observe that $\varepsilon^{2} \bar{\gamma}^{2}+\delta^{2}<\alpha^{2}$ for $\varepsilon>0$ close enough to 0 ). To prove the claim, we use an argument similar to the one used in the proof of the Linear Decomposition Lemma (i.e. Lemma 4.2).

To begin with, since the problem is linear, we may as well assume that

$$
\|w\|_{L_{-\bar{\gamma} \varepsilon,-\delta}^{2}\left(\mathbb{R}^{2}\right)}=1 .
$$

Then, elliptic estimates imply that $u \in W_{-\bar{\gamma} \varepsilon,-\delta}^{2,2}\left(\mathbb{R}^{2}\right)$ and

$$
\|w\|_{W_{-\bar{\gamma} \varepsilon,-\delta}^{2,2}\left(\mathbb{R}^{2}\right)} \leq C\|w\|_{L_{-\bar{\gamma} \varepsilon,-\delta}^{2}\left(\mathbb{R}^{2}\right)} .
$$

For $j=1, \ldots, k$, we define $\hat{\mathbb{I}}_{j}$ to be smooth cutoff functions such that

$$
\operatorname{supp} \hat{\mathbb{I}}_{j} \subset\left\{\mathrm{x} \in \mathbb{R}^{2}: \operatorname{dist}\left(\mathrm{x}, \mathcal{C}_{j, \varepsilon}\right) \leq \operatorname{dist}\left(\mathrm{x}, \mathcal{C}_{i, \varepsilon}\right)-\frac{\sqrt{2}}{4} \log \varepsilon, \quad \forall i \neq j\right\},
$$

and $\hat{\mathbb{I}}_{j}(\mathrm{x}) \equiv 1$ when $\operatorname{dist}\left(\mathrm{x}, \mathcal{C}_{j, \varepsilon}\right) \leq \operatorname{dist}\left(\mathrm{x}, \mathcal{C}_{i, \varepsilon}\right)$ for $i \neq j$. Thanks to (9.7), we know that

$$
\left\|\hat{\mathbb{I}}_{j_{0}} w\right\|_{L_{-\bar{\gamma} \varepsilon,-\delta}^{2}\left(\mathbb{R}^{2}\right)} \geq \frac{1}{2 k},
$$

for some $j_{0} \in\{1, \ldots, k\}$. We define $w_{0}:=\hat{\mathbb{I}}_{j_{0}} w$. Observe that

$$
\mathfrak{L}_{\varepsilon} w_{0}=\left[\Delta, \hat{\mathbb{I}}_{j_{0}}\right] w
$$

where $\mathfrak{L}_{\varepsilon}:=-\Delta+F^{\prime \prime}\left(u_{\varepsilon}\right)$.

Let $(t, s)$ be Fermi coordinates associated to $\mathcal{C}_{j_{0}, \varepsilon}$, so that $s$ is the arc length on $\mathcal{C}_{j_{0}, \varepsilon}$ and $t(\mathrm{x}):=\operatorname{dist}^{s}\left(\mathrm{x}, \mathcal{C}_{j_{0}, \varepsilon}\right)$ denotes the signed distance to $\mathcal{C}_{j_{0}, \varepsilon}$. We change the coordinates from $(x, y)$ to Fermi coordinates $(t, s)$ (this change of variables is quite standard and for details, we refer the reader to calculations in Section 5 of [8]).

Using (9.3), (9.6) together with the assumption on the function $w$, we check that

$$
h_{\varepsilon}:=-\left(\partial_{s}^{2}+\partial_{t}^{2}-F^{\prime \prime}\left(u_{0}(t)\right)\right) w_{0}
$$

satisfies

$$
\left\|h_{\varepsilon}\right\|_{\mathbb{L}_{-\bar{\gamma} \varepsilon,-\delta}^{2}\left(\mathbb{R}^{2}\right)} \leq C\left(\varepsilon^{\tau}-\frac{1}{\log \varepsilon}\right)\|w\|_{L_{-\bar{\gamma} \varepsilon,-\delta}^{2}\left(\mathbb{R}^{2}\right)},
$$

for some $\tau>0$. Thanks to the Linear Decomposition Lemma (Lemma 4.2), we know that $w_{0} \in \mathbb{L}_{-\bar{\gamma} \varepsilon,-\delta}^{2}\left(\mathbb{R}^{2}\right)$ and also that

$$
\left\|w_{0}\right\|_{\mathbb{L}_{-\bar{\gamma} \varepsilon,-\delta}^{2}\left(\mathbb{R}^{2}\right)} \leq C\left(\varepsilon^{\tau}-\frac{1}{\log \varepsilon}\right)\|w\|_{L_{-\bar{\gamma} \varepsilon,-\delta}^{2}\left(\mathbb{R}^{2}\right)} .
$$

For $\varepsilon$ small enough this inequality is not compatible with (9.8) and (9.7). Having reached a contradiction, we conclude that $w_{0} \equiv 0$ and hence $w \equiv 0$. This completes the proof of the result. 


\section{The Lagrangian structure and the proof of Theorem 2.3}

We assume that we are given a function $u \in \mathcal{M}_{2 k}$ which is nondegenerate. The tangent space $T_{u} \mathcal{M}_{2 k}$ is spanned by the functions $w$ which satisfy

$$
\mathfrak{L} w=0,
$$

where $\mathfrak{L}$ is the linearized operator about $u$. According to the result of Proposition 6.1 and Proposition 6.2 we know that such a function $w$ can be decomposed into

$$
w=\sum_{j=1}^{2 k} a_{j} \mathrm{~d} u\left(X_{j}\right)+b_{j} \mathrm{~d} u\left(Y_{j}\right)+\bar{w},
$$

where $a_{j}, b_{j} \in \mathbb{R}$ and $\bar{w} \in W_{-\gamma,-\delta}^{2,2}\left(\mathbb{R}^{2}\right)$ for some $\gamma, \delta>0$. Let us consider two such functions $w^{(1)}, w^{(2)} \in T_{u} \mathcal{M}_{2 k}$ (the coefficients and functions in the corresponding decompositions are adorned with superscripts (1) and (2)). For all $R>0$, we integrate $\left(w^{(1)} \mathfrak{L} w^{(2)}-w^{(2)} \mathfrak{L} w^{(1)}\right)$ over the ball of radius $R$ to get

$$
0=\int_{B_{R}}\left(w^{(1)} \mathfrak{L} w^{(2)}-w^{(2)} \mathfrak{L} w^{(1)}\right) d \mathrm{x}=\int_{\partial B_{R}}\left(w^{(1)} \partial_{r} w^{(2)}-w^{(2)} \partial_{r} w^{(1)}\right) d s .
$$

But, using (10.1) and letting $R \longrightarrow \infty$, we conclude without any difficulty that

$$
\sum_{j=1}^{2 k}\left(a_{j}^{(1)} b_{j}^{(2)}-a_{j}^{(2)} b_{j}^{(1)}\right)=0 .
$$

This completes the proof of Theorem 2.3

\section{Appendix A}

Assume that $u$ is a solution of (2.1) which is defined in $\mathbb{R}^{2}$. Assume that $X$ and $Y$ are two vector fields also defined in $\mathbb{R}^{2}$. In coordinates, we can write

$$
X=\sum_{j} X^{j} \partial_{x_{j}}, \quad Y=\sum_{j} Y^{j} \partial_{x_{j}},
$$

and, if $f$ is a smooth function, we use the following notation:

$$
\begin{gathered}
X(f):=\sum_{j} X^{j} \partial_{x_{j}} f, \quad \nabla f:=\sum_{j} \partial_{x_{j}} f \partial_{x_{j}}, \\
\operatorname{div} X:=\sum_{i} \partial_{x_{i}} X^{i},
\end{gathered}
$$

and

so that

$$
\mathrm{d}^{*} X:=\frac{1}{2} \sum_{i, j}\left(\partial_{x_{i}} X^{j}+\partial_{x_{j}} X^{i}\right) \mathrm{d} x_{i} \otimes \mathrm{d} x_{j}
$$

We claim that:

$$
\mathrm{d}^{*} X(Y, Y)=\sum_{i, j} \partial_{x_{i}} X^{j} Y^{i} Y^{j}
$$

Lemma 11.1 (Balancing formula). The following identity holds:

$\operatorname{div}\left(\left(\frac{1}{2}|\nabla u|^{2}+F(u)\right) X-X(u) \nabla u\right)=\left(\frac{1}{2}|\nabla u|^{2}+F(u)\right) \operatorname{div} X-\mathrm{d}^{*} X(\nabla u, \nabla u)$. 
Proof. This follows from a direct computation.

Translations of $\mathbb{R}^{2}$ correspond to the constant vector field

$$
X:=X_{0},
$$

where $X_{0}$ is a fixed vector, while rotations correspond to the vector field

$$
X:=x \partial_{y}-y \partial_{x} .
$$

In either case, we have $\operatorname{div} X=0$ and $\mathrm{d}^{*} X=0$. Therefore, we conclude that

$$
\operatorname{div}\left(\left(\frac{1}{2}|\nabla u|^{2}+F(u)\right) X-X(u) \nabla u\right)=0,
$$

for these two vector fields. The divergence theorem implies that

$$
\int_{\partial \Omega}\left(\left(\frac{1}{2}|\nabla u|^{2}+F(u)\right) X-X(u) \nabla u\right) \cdot \nu d s=0,
$$

where $\nu$ is the (outward-pointing) unit normal vector field to $\partial \Omega$.

We define

$$
c_{0}:=\int_{-\infty}^{+\infty}\left(\frac{1}{2}\left(\partial_{x} u_{0}\right)^{2}+F\left(u_{0}\right)\right) d x
$$

which only depends on the potential $F$. We now assume that $u \in \mathcal{M}_{2 k}$ and we keep the notation introduced in $\S 2$. We use (11.1) with $\Omega$, which is equal to the ball of radius $R$ centered at the origin, and then we let $R$ tend to $\infty$. Taking $X=X_{0}$ and using (1.4), we find with little work that

$$
c_{0} \sum_{j=1}^{2 k} \mathrm{e}_{j} \cdot X_{0}=0
$$

Taking $X=x \partial_{y}-y \partial_{x}$, we find that

$$
c_{0} \sum_{j=1}^{2 k} r_{j}=0
$$

In other words,

$$
\sum_{j=1}^{2 k} r_{j}=0
$$

This completes the proof of (2.16) and (2.17).

Remark 11.1. These conservation laws stem from the fact that (2.1) is invariant under the action of isometries of $\mathbb{R}^{2}$.

\section{Appendix B}

We keep the notation introduced in the proof of Proposition 3.1, and we define

$$
\hat{W}(x):=\frac{1}{\mathbb{W}_{\zeta}} W_{\zeta}^{-}(x) \int_{-\infty}^{x} W_{\zeta}^{+}(y) V(y) d y .
$$

Since $\left|W_{\zeta}^{ \pm}\right| \leq C e^{ \pm \delta_{\zeta} x}$, we can write

$$
|\hat{W}(x)| \leq C \tilde{W}(x)
$$


where

$$
\tilde{W}(x):=e^{-\delta_{\zeta} x} \int_{-\infty}^{x} e^{\delta_{\zeta} y}|V(y)| d y .
$$

Obviously, it is enough to prove that

$$
\|\tilde{W}\|_{\tilde{L}_{\delta}^{2}(\mathbb{R}, \mathbb{C})} \leq C\|V\|_{\tilde{L}_{\delta}^{2}(\mathbb{R}, \mathbb{C})},
$$

since this immediately implies that

$$
\|\hat{W}\|_{\tilde{L}_{\delta}^{2}(\mathbb{R}, \mathbb{C})} \leq C\|V\|_{\tilde{L}_{\delta}^{2}(\mathbb{R}, \mathbb{C})} .
$$

The result then follows from this estimate, together with a similar estimate for the function

$$
\check{W}(x):=\frac{1}{W_{\zeta}} W_{\zeta}^{+}(x) \int_{x}^{+\infty} W_{\zeta}^{-}(y) V(y) d y .
$$

To prove (12.1), we argue as follows. First, the following pointwise estimate follows from the Cauchy-Schwarz inequality

$$
\left|(\cosh y)^{-\delta} \tilde{W}(y)\right| \leq c\|V\|_{\tilde{L}_{\delta}^{2}(\mathbb{R}, \mathbb{C})},
$$

for all $y \in \mathbb{R}$. Next, we estimate

$$
\begin{aligned}
\int_{-\infty}^{0} e^{2 \delta x} \tilde{W}^{2}(x) d x= & \int_{-\infty}^{0} e^{2\left(\delta-\delta_{\zeta}\right) x}\left(\int_{-\infty}^{x} e^{\delta_{\zeta} y}|V(y)| d y\right)^{2} d x \\
= & \lim _{s \rightarrow-\infty}\left[\frac{1}{2\left(\delta-\delta_{\zeta}\right)} e^{2 \delta x} \tilde{W}^{2}(y)\right]_{s}^{0} \\
& -\frac{1}{\delta-\delta_{\zeta}} \int_{-\infty}^{0} e^{2 \delta x} V(x) \tilde{W}(x) d x \\
\leq & C\left(\|V\|_{\tilde{L}_{\delta}^{2}(\mathbb{R}, \mathbb{C})}^{2}+\|\tilde{W}\|_{\tilde{L}_{\delta}^{2}(\mathbb{R}, \mathbb{C})}\right)\|V\|_{\tilde{L}_{\delta}^{2}(\mathbb{R}, \mathbb{C})},
\end{aligned}
$$

where we have used (12.2) together with the Cauchy-Schwarz inequality in order to obtain the last estimate. Using similar arguments, we also get

$$
\int_{0}^{+\infty} e^{-2 \delta x} \tilde{W}^{2}(x) d x \leq C\left(\|V\|_{\tilde{L}_{\delta}^{2}(\mathbb{R}, \mathbb{C})}^{2}+\|\tilde{W}\|_{\tilde{L}_{\delta}^{2}(\mathbb{R}, \mathbb{C})}\|V\|_{\tilde{L}_{\delta}^{2}(\mathbb{R}, \mathbb{C})}\right) .
$$

Hence, we conclude that

$$
\|\tilde{W}\|_{\tilde{L}_{\delta}^{2}(\mathbb{R}, \mathbb{C})}^{2} \leq C\left(\|V\|_{\tilde{L}_{\delta}^{2}(\mathbb{R}, \mathbb{C})}^{2}+\|\tilde{W}\|_{\tilde{L}_{\delta}^{2}(\mathbb{R}, \mathbb{C})}\|V\|_{\tilde{L}_{\delta}^{2}(\mathbb{R}, \mathbb{C})}\right)
$$

which implies that

$$
\|\tilde{W}\|_{\tilde{L}_{\delta}^{2}(\mathbb{R}, \mathbb{C})}^{2} \leq C\|V\|_{\tilde{L}_{\delta}^{2}(\mathbb{R}, \mathbb{C})}^{2} .
$$

This completes the proof of the estimate.

\section{ACKNOWLEDGMENTS}

The authors would like to thank the referee for some important remarks which have been very helpful in improving the exposition of the paper. This work has been partly supported by Chilean research grants Fondecyt 1070389, 1090103, Fondo Basal CMM-Chile, Project Añillo ACT-125 CAPDE, an Ecos-Conicyt contract and a grant under the MathAmSud program. The third author was partially supported by the ANR-08-BLANC-0335-01 grant. 


\section{REFERENCES}

1. M. Adler, On a trace functional for formal pseudo differential operators and the symplectic structure of the Korteweg-de Vries type equations, Invent. Math. 50 (1978/79), no. 3, 219-248. MR520927 (80i:58026)

2. Francesca Alessio, Alessandro Calamai, and Piero Montecchiari, Saddle-type solutions for a class of semilinear elliptic equations, Adv. Differential Equations 12 (2007), no. 4, 361-380. MR2305872 (2007m:35051)

3. Nicholas D. Alikakos, Giorgio Fusco, and Vagelis Stefanopoulos, Critical spectrum and stability of interfaces for a class of reaction-diffusion equations, J. Differential Equations 126 (1996), no. 1, 106-167. MR 1382059 (97d:35100)

4. Haïm Brezis, Analyse fonctionnelle, Collection Mathématiques Appliquées pour la Maîtrise. [Collection of Applied Mathematics for the Master's Degree], Masson, Paris, 1983, Théorie et applications. [Theory and applications]. MR697382(85a:46001)

5. Xavier Cabré and Joana Terra, Saddle-shaped solutions of bistable diffusion equations in all of $\mathbb{R}^{2 m}$, J. Eur. Math. Soc. (JEMS) 11 (2009), no. 4, 819-843. MR2538506

6. Earl A. Coddington and Norman Levinson, Theory of ordinary differential equations, McGraw-Hill Book Company, Inc., New York-Toronto-London, 1955. MR0069338 (16,1022b)

7. Ha Dang, Paul C. Fife, and L. A. Peletier, Saddle solutions of the bistable diffusion equation, Z. Angew. Math. Phys. 43 (1992), no. 6, 984-998. MR1198672 (94b:35041)

8. Manuel del Pino, Michał Kowalczyk, Frank Pacard, and Juncheng Wei, Multiple-end solutions to the Allen-Cahn equation in $\mathbb{R}^{2}$, J. Funct. Anal. 258 (2010), no. 2, 458-503. MR2557944

9. N. Ghoussoub and C. Gui, On a conjecture of De Giorgi and some related problems, Math. Ann. 311 (1998), no. 3, 481-491. MR1637919 (99j:35049)

10. Changfeng Gui, Hamiltonian identities for elliptic partial differential equations, J. Funct. Anal. 254 (2008), no. 4, 904-933. MR2381198(2009b:35112)

11. __ Even symmetry of some entire solutions to the Allen-Cahn equation in two dimensions, Preprint (2011).

12. Nick Korevaar, Rafe Mazzeo, Frank Pacard, and Richard Schoen, Refined asymptotics for constant scalar curvature metrics with isolated singularities, Invent. Math. 135 (1999), no. 2, 233-272. MR1666838 (2001a:35055)

13. Bertram Kostant, The solution to a generalized Toda lattice and representation theory, Adv. in Math. 34 (1979), no. 3, 195-338. MR.550790 (82f:58045)

14. Michał Kowalczyk and Yong Liu, Nondegeneracy of the saddle solution of the Allen-Cahn equation on the plane, Proc. Amer. Math. Soc. 139 (2011), no. 12, 4319-4329. MR 2823077 (2012h:35185)

15. R. Kusner, R. Mazzeo, and D. Pollack, The moduli space of complete embedded constant mean curvature surfaces, Geom. Funct. Anal. 6 (1996), no. 1, 120-137. MR1371233 (97b:58022)

16. Robert B. Lockhart and Robert C. McOwen, Elliptic differential operators on noncompact manifolds, Ann. Scuola Norm. Sup. Pisa Cl. Sci. (4) 12 (1985), no. 3, 409-447. MR837256 (87k:58266)

17. Rafe Mazzeo and Daniel Pollack, Gluing and moduli for noncompact geometric problems, Geometric theory of singular phenomena in partial differential equations (Cortona, 1995), Sympos. Math., XXXVIII, Cambridge Univ. Press, Cambridge, 1998, pp. 17-51. MR 1702086 (2000i:53058)

18. Rafe Mazzeo, Daniel Pollack, and Karen Uhlenbeck, Moduli spaces of singular Yamabe metrics, J. Amer. Math. Soc. 9 (1996), no. 2, 303-344. MR.1356375 (96f:53055)

19. Jürgen Moser, Finitely many mass points on the line under the influence of an exponential potential-an integrable system, Dynamical systems, theory and applications (Rencontres, BattelleRes. Inst., Seattle, Wash., 1974), Springer, Berlin, 1975, pp. 467-497. Lecture Notes in Phys., Vol. 38. MR0455038 (56:13279)

20. Arnold F. Nikiforov and Vasilii B. Uvarov, Special functions of mathematical physics, A unified introduction with applications, Translated from the Russian and with a preface by Ralph P. Boas, With a foreword by A. A. Samarskiı̌. Birkhäuser Verlag, Basel, 1988. MR922041 (89h:33001)

21. M. A. Olshanetsky and A. M. Perelomov, Explicit solutions of classical generalized Toda models, Invent. Math. 54 (1979), no. 3, 261-269. MR553222 (80m:58018) 
22. Frank Pacard, Connected sum contruction in geometry and nonlinear analysis, Unpublished lecture notes, 2008.

23. M. Schatzman, On the stability of the saddle solution of Allen-Cahn's equation, Proc. Roy. Soc. Edinburgh Sect. A 125 (1995), no. 6, 1241-1275. MR1363002 (96j:35085)

24. W. W. Symes, Systems of Toda type, inverse spectral problems, and representation theory, Invent. Math. 59 (1980), no. 1, 13-51. MR575079 (81g:58019)

Departamento de Ingeniería Matemática and Centro de Modelamiento Matemático (UMi 2807 CNRS), Universidad de Chile, Casilla 170 Correo 3, Santiago, Chile

E-mail address: delpino@dim.uchile.cl

Departamento de Ingeniería Matemática and Centro de Modelamiento Matemático (UMi 2807 CNRS), Universidad de Chile, Casilla 170 Correo 3, Santiago, Chile

E-mail address: kowalczy@dim.uchile.cl

Centre de Mathématiques Laurent Schwartz UMR-CNRS 7640, École Polytechnique, 91128 Palaiseau, France

E-mail address: frank.pacard@math.polytechnique.fr 